\title{
ALGEBRAIC CYCLES AND MUMFORD-GRIFFITHS INVARIANTS
}

\author{
James D. Lewis ${ }^{\dagger}$ and Shuji Saito
}

May 2007

\begin{abstract}
Let $X$ be a projective algebraic manifold and let $\mathrm{CH}^{r}(X)$ be the Chow group of algebraic cycles of codimension $r$ on $X$, modulo rational equivalence. Working with a candidate Bloch-Beilinson filtration $\left\{F^{\nu}\right\}_{\nu \geq 0}$ on $\mathrm{CH}^{r}(X) \otimes \mathbb{Q}$ due to the second author, we construct a space of arithmetic Hodge theoretic invariants $\nabla J^{r, \nu}(\bar{X})$ and corresponding map $\phi_{X}^{r, \nu}: G r_{F}^{\nu} \mathrm{CH}^{r}(X) \otimes \mathbb{Q} \rightarrow \nabla J^{r, \nu}(X)$, and determine conditions on $X$ for which the kernel and image of $\phi_{X}^{r, \nu}$ are "uncountably large".
\end{abstract}

\section{CONTENTS}

$\S 0$. Introduction

$\S 1$. Notation

$\S 2$. Good filtrations

$\S 3$. Arithmetic de Rham cohomology

$\S 4$. Capturing nontrivial classes via $\phi_{X}^{r, \nu}$

$\S 5$. Cycle classes in higher extension groups

§6. A map from cycle classes to de Rham/Mumford-Griffiths invariants

§7. Detecting nonzero classes with trivial de Rham/Mumford-Griffiths invariant

$\S 8$. A class of examples with trivial Mumford-Griffiths invariant

Appendix: A conjectural overview

References

\section{$\S 0$. InTRODUCTION}

Let $X$ be a projective smooth variety over $\mathbb{C}$ and let $\mathrm{CH}^{r}(X)$ be the Chow group of algebraic cycles of codimension $r$ on $X$ modulo rational equivalence. A fundamental problem is to search for a reasonable set of invariants (e.g. Hodge theoretic) that provides us with a good understanding of the structure of $\mathrm{CH}^{r}(X)$. The first significant step towards this problem was taken by Griffiths (1969) who defined Abel-Jacobi maps

$$
\rho_{X}^{r}: \mathrm{CH}_{\mathrm{hom}}^{r}(X) \rightarrow J^{r}(X)
$$

where $\mathrm{CH}_{\text {hom }}^{r}(X)=\operatorname{ker}\left\{\mathrm{CH}^{r}(X) \rightarrow H^{2 r}(X, \mathbb{Z})\right\}$ is the subgroup of those cycle classes that are homologically equivalent to zero, and where $J^{r}(X)$ is the $r$-th intermediate Jacobian of $X$ in the sense of Griffiths [Gri]. If the Griffiths Abel-Jacobi map were an isomorphism, then there would not be much to explore in the world of algebraic cycles. That the map is not surjective follows from the work of Griffiths (op. cit.), using

1991 Mathematics Subject Classification. 14C25, 14C30.

Key words and phrases. Mumford-Griffiths invariant, algebraic cycle, Bloch-Beilinson filtration, Chow group.

${ }^{\dagger}$ Partially supported by a grant from the Natural Sciences and Engineering Research Council of Canada. 
Hodge theory and monodromy arguments; that the kernel is far from injective in general is a consequence of Mumford's seminal work [Mu].

An important observation is the following natural isomorphism discovered by Carlson [Ca],

$$
J^{r}(X) \simeq \operatorname{Ext}_{\mathrm{MHS}}^{1}\left(\mathbb{Z}, H^{2 r-1}(X, \mathbb{Z}(r))\right),
$$

where MHS denotes the category of graded polarizable $\mathbb{Q}$-mixed Hodge structures introduced by Deligne [De1]. This implies that an element of $\mathrm{CH}_{\mathrm{hom}}^{r}(X) / \operatorname{ker}\left(\rho_{X}^{r}\right)$ is detected by an extension in MHS. One may then have a naive expectation that there may be a secondary cycle class map from $\operatorname{ker}\left(\rho_{X}^{r}\right)$ to higher extension groups $\operatorname{Ext}_{\mathrm{MHS}}^{p}$, which fails due to the fact that $\operatorname{Ext}_{\mathrm{MHS}}^{p}=0$ for $p \geq 2$ ([Bei] (Cor. 1.10)). (Note that $\operatorname{Ext}_{\mathrm{MHS}}^{p}=0$ for $p \geq 2$ also follows from the fact that $\operatorname{Ext}_{\mathrm{MHS}}^{1}(A,-)$ is exact, using Carlson's explicit description of $\operatorname{Ext}_{\mathrm{MHS}}^{1}$ ([Ca]. See also [As1] 2.5.) It was A. Beilinson who had an innovative idea to remedy the situation. He postulated the existence of a category (called the category of mixed motives) whose higher extension groups capture all elements of Chow groups. A more precise formulation is the following conjecture. In this paper we only consider Chow groups with rational coefficients:

$$
\mathrm{CH}^{r}(X ; \mathbb{Q})=\mathrm{CH}^{r}(X) \otimes \mathbb{Q} .
$$

Conjecture 0.1. For every projective smooth variety $X$ over $\mathbb{C}$, there exists a canonical and functorial filtration (called the Bloch-Beilinson filtration)

$$
C H^{r}(X ; \mathbb{Q})=F^{0} C H^{r}(X ; \mathbb{Q}) \supset F^{1} C H^{r}(X ; \mathbb{Q}) \supset F^{2} C H^{r}(X ; \mathbb{Q}) \supset \cdots
$$

such that the following formula holds for each integer $\nu \geq 0$ :

$$
F^{\nu} C H^{r}(X ; \mathbb{Q}) / F^{\nu+1} C H^{r}(X ; \mathbb{Q}) \simeq \operatorname{Ext}_{\mathcal{M M}}^{\nu}\left(1, h^{2 r-\nu}(X)(r)\right) .
$$

Here $\mathcal{M M}$ denotes the (still conjectural) category of mixed motives over $\mathbb{C}$ which contains as a full subcategory Grothendieck's category $\mathcal{M}$ of (pure) motives over $\mathbb{C}, h^{*}(X)(r) \in \mathcal{M}$ denotes the cohomological object with Tate twist associated to $X$ and $1=h^{0}(\operatorname{Spec}(\mathbb{C}))$.

In [Ja] and [Sa2] it is proven that the Bloch-Beilinson filtration is unique if it exists under the assumption of Grothendieck's standard conjectures. Several candidates for the Bloch-Beilinson filtration have been proposed. From these we adopt the filtration

$$
F_{B}^{\nu} \mathrm{CH}^{r}(X ; \mathbb{Q}) \subset \mathrm{CH}^{r}(X ; \mathbb{Q}) \quad(\nu \geq 0)
$$

defined in [Sa2], Def.(1-3). We recall the definition of this filtration in $\S 2$.

The main results of this paper can be explained as follows. We introduce the spaces $\nabla J^{r, \nu}(X)$ of MumfordGriffiths invariants in $\S 3$, which are defined in terms of arithmetic de Rham cohomology. It is given by the cohomology of the complex

$$
\Omega_{\mathbb{C} / \mathbb{Q}}^{\nu-1} \otimes F^{r-\nu+1} H_{\mathrm{DR}}^{2 r-\nu}(X / \mathbb{C}) \stackrel{\nabla}{\rightarrow} \Omega_{\mathbb{C} / \overline{\mathbb{Q}}}^{\nu} \otimes F^{r-\nu} H_{\mathrm{DR}}^{2 r-\nu}(X / \mathbb{C}) \stackrel{\nabla}{\longrightarrow} \Omega_{\mathbb{C} / \mathbb{Q}}^{\nu+1} \otimes F^{r-\nu-1} H_{\mathrm{DR}}^{2 r-\nu}(X / \mathbb{C}),
$$

where $H_{\mathrm{DR}}^{q}(X / \mathbb{C})$ is the de Rham cohomology of $X / \mathbb{C}$ with the Hodge filtration $F^{p} H_{\mathrm{DR}}^{q}(X / \mathbb{C})$ and $\nabla$ is the arithmetic Gauss-Manin connection. Then there is a cycle map (Proposition 3.7)

$$
\phi_{X}^{r, \nu}: G r_{F_{B}}^{\nu} \mathrm{CH}^{r}(X ; \mathbb{Q}) \rightarrow \nabla J^{r, \nu}(X) .
$$

By "forgetting" the Hodge filtration, we also have a map

$$
\phi_{X, D R}^{r, \nu}: G r_{F_{B}}^{\nu} \mathrm{CH}^{r}(X ; \mathbb{Q}) \rightarrow \nabla D R^{r, \nu}(X),
$$

where $\nabla D R^{r, \nu}(X)$ is the coarser space of de Rham invariants given by the cohomology of the complex

$$
\Omega_{\mathbb{C} / \overline{\mathbb{Q}}}^{\nu-1} \otimes H_{\mathrm{DR}}^{2 r-\nu}(X / \mathbb{C}) \stackrel{\nabla}{\rightarrow} \Omega_{\mathbb{C} / \overline{\mathbb{Q}}}^{\nu} \otimes H_{\mathrm{DR}}^{2 r-\nu}(X / \mathbb{C}) \stackrel{\nabla}{\rightarrow} \Omega_{\mathbb{C} / \mathbb{Q}}^{\nu+1} \otimes H_{\mathrm{DR}}^{2 r-\nu}(X / \mathbb{C}) .
$$

The first main result affirms that under various assumptions on $X$, the image of $\phi_{X, D R}^{r, \nu}$ is large, where "large" has a similar meaning to that in the following theorem of Mumford [Mu]: 
Theorem. Let $X$ be a projective smooth surface over $\mathbb{C}$. Let $A_{0}(X) \subset C H_{0}(X ; \mathbb{Q})$ denote the subgroup of the classes of zero-cycles of degree zero. Assume $H^{0}\left(X, \Omega_{X / \mathbb{C}}^{2}\right) \neq 0$. Then $A_{0}(X)$ is infinite dimensional, viz., it is impossible to find $Y_{1}, \ldots, Y_{N}$, proper smooth connected curves with a morphism $f: Y:=\coprod_{i=1}^{N} Y_{i} \rightarrow X$ such that $f_{*}: A_{0}(Y) \rightarrow A_{0}(X)$ is surjective.

We will give the following refinement of the above result:

Theorem 0.2. Let $X$ be a projective smooth variety of dimension d over $\mathbb{C}$ and consider

$$
\phi_{X, D R}^{d, \nu}: F_{B}^{\nu} C H_{0}(X ; \mathbb{Q}) \rightarrow \nabla D R^{d, \nu}(X) .
$$

Assume that there exists a dominant rational map $\pi: \widetilde{X} \rightarrow X$ such that the hard Lefschetz conjecture $B(\widetilde{X})$ for $\tilde{X}$ holds (see Notation $(v))$. Assume further that $H^{0}\left(X, \Omega_{X / \mathbb{C}}^{\nu}\right) \neq 0$ for an integer $\nu \geq 2$. Then it is impossible to find $\left\{f_{i}: Y_{i} \rightarrow X\right\}_{i \in \mathbb{N}}$, a countable set of morphisms of proper smooth varieties over $\mathbb{C}$ such that $\operatorname{dim}\left(Y_{i}\right) \leq \nu-1$ and

$$
F_{B}^{\nu} C H_{0}(X ; \mathbb{Q}) \subset \text { Image }\left\{\bigoplus_{i \in \mathbb{N}} A_{0}\left(Y_{i}\right) \stackrel{f_{*}}{\longrightarrow} A_{0}(X)\right\}+\operatorname{ker}\left(\phi_{X, D R}^{d, \nu}\right),
$$

where $f_{*}$ is induced by $f_{i}$ for $i \in \mathbb{N}$.

We recall that for a smooth projective variety $W, B(W)$ is known to hold if $W$ is obtained under successive operations of products and hypersurface sections starting from curves, surfaces, Abelian varieties, smooth complete intersections.

Our next result is an analogue of Theorem 0.2 for algebraic equivalence on a hypersurface. By a general hypersurface $X$ of dimension $d$ and of degree $m$, we mean a hypersurface corresponding to a point in a certain nonempty Zariski open subset of the universal family of hypersurfaces of degree $m$ in $\mathbb{P}^{d+1}$.

Theorem 0.3. Let $X \subset \mathbb{P}^{d+1}$ be a smooth hypersurface of degree $m \geq 3$ over $\mathbb{C}$. Put

$$
k=\left[\frac{d+1}{m}\right] \quad \text { and } \quad r=d-k, \nu=2 r-d=d-2 k
$$

and assume $k \geq 1, \nu \geq 2$, and the numerical condition:

$$
k(d+2-k)+1-\left(\begin{array}{c}
m+k \\
k
\end{array}\right) \geq 0 .
$$

Consider

$$
\phi_{X, D R}^{r, \nu}: F_{B}^{\nu} C H^{r}(X ; \mathbb{Q}) \rightarrow \nabla D R^{r, \nu}(X) .
$$

Assume that $X$ is general. Then it is impossible to find $\left\{f_{i}: Y_{i} \rightarrow X\right\}_{i \in \mathbb{N}}$, a countable set of morphisms of proper smooth varieties over $\mathbb{C}$ such that $\operatorname{dim}\left(Y_{i}\right) \leq k+\nu-1$ and

$$
A^{r}(X) \subset \text { Image }\left\{\bigoplus_{i \in \mathbb{N}} A_{k}\left(Y_{i}\right) \stackrel{f_{*}}{\longrightarrow} A^{r}(X)\right\}+\operatorname{ker}\left(\phi_{X, D R}^{r, \nu}\right),
$$

where $f_{*}$ is induced by $f_{i}$ for $i \in \mathbb{N}$. Here $A^{r}(X) \subset C H_{\mathrm{hom}}^{r}(X ; \mathbb{Q})$ denotes the subgroup of the cycle classes algebraically equivalent to zero.

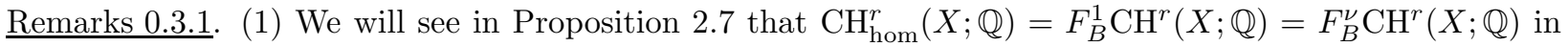
Theorem 0.3. 
(2) The hypersurfaces in question in Theorem 0.3, albeit very interesting geometrically, are of a low degree. In particular, since $k \geq 1$ it follows that $H^{0}\left(X, \Omega_{X / \mathbb{C}}^{d}\right)=0$.

For the proof of the above results, we will introduce an integral invariant $r k(\Xi)$ for $\Xi \subset A^{r}(X)$, called the rank of $\Xi$ modulo $\operatorname{ker}\left(\phi_{X, D R}^{r, \nu}\right)$, which measures the "size" of the image of $\Xi \cap F_{B}^{\nu} \mathrm{CH}^{r}(X ; \mathbb{Q})$ under $\phi_{X, D R}^{r, \nu}$. The main technical result in this direction is stated in Theorem 4.2, whose proof is based on an arithmetic version of Salberger's duality pairing, and the essential ideas rely on the constructions in [Sa1]. By working with the coarser de Rham invariants, we arrive at similar statements for the Mumford-Griffiths invariants.

With regard to $\operatorname{ker}\left(\phi_{X}^{r, \nu}\right)$, if $X=X_{o} \times \overline{\mathbb{Q}} \mathbb{C}$ with $X_{o}$ smooth projective over $\overline{\mathbb{Q}}$, we introduce arithmetic Hodge theoretic invariants $H_{\overline{\mathbb{Q}}}^{r-\nu, r}\left(X_{o} / \overline{\mathbb{Q}}\right) \subset H^{r}\left(X, \Omega_{X / \mathbb{C}}^{r-\nu}\right)$ which serve as an obstruction to countability of $\operatorname{ker}\left(\phi_{X}^{r, \nu}\right)$. We will deduce the following:

Theorem 0.4. Let $X_{o}$ be a smooth projective variety over $\overline{\mathbb{Q}}$ and $X=X_{o} \times_{\mathbb{Q}} \mathbb{C}$. Assume that the Künneth components of the diagonal class of $X$ are algebraic. If $\nu \geq 2$ and if $H_{\overline{\mathbb{Q}}}^{r-\nu, r}\left(X_{o} / \overline{\mathbb{Q}}\right) \neq 0$, then there are an uncountable number of classes in the kernel of $\phi_{X}^{r, \nu}$.

We will show how to construct a class of examples for which the assumptions of Theorem 0.4 are satisfied. The examples arise from products involving 1-motives over $\overline{\mathbb{Q}}$, in particular the product of smooth projective curves defined over $\overline{\mathbb{Q}}$, and an Abelian variety defined over $\overline{\mathbb{Q}}$. The precise statements of the main results appear in $\S 7$ and $\S 8$. We believe that these theorems provide the strongest results to date on the properties of $\phi_{X}^{r, \nu}$. For instance, Theorem 0.2 generalizes and strengthens Theorem 4.1 in [MSa2]. We note that it was Asakura ([As2]) who first found an example of a nontrivial cycle with trivial Mumford-Griffiths invariant.

To provide the reader with a better understanding of the results of this paper, we briefly discuss the technique of taking a $\overline{\mathbb{Q}}$-spread. This technique abounds in a number of works (e.g. [Gr-Gr], [Le2], [MSa1-2], [As1]). The basic idea of the construction is the following. Given $X$, smooth projective over $\mathbb{C}$, we can find a $\overline{\mathbb{Q}}$-spread, namely a smooth affine variety $S$ over $\overline{\mathbb{Q}}$ with

$$
f: \mathcal{X}_{S} \rightarrow S
$$

a projective smooth morphism of varieties over $\overline{\mathbb{Q}}$, with a morphism $\eta: \operatorname{Spec}(\mathbb{C}) \rightarrow S$ whose image is the generic point of $S$, such that $\mathcal{X}_{S} \times{ }_{S} \operatorname{Spec}(\mathbb{C})$, the base change via $\eta$, is isomorphic to $X$. Similarly, if $\xi \in \mathrm{CH}^{r}(X ; \mathbb{Q})$ is given, then after making a base change if necessary, $\xi$ has a lifting $\tilde{\xi} \in \mathrm{CH}^{r}\left(\mathcal{X}_{S} ; \mathbb{Q}\right)$. We then have the following variational version of $\phi_{X}^{r, \nu}$ :

$$
\phi_{\mathcal{X}_{S} / S}^{r, \nu}: F_{B}^{\nu} \mathrm{CH}^{r}\left(\mathcal{X}_{S} / S ; \mathbb{Q}\right) \rightarrow \nabla J^{r, \nu}\left(\mathcal{X}_{S} / S\right)
$$

We may retrieve $\phi_{X}^{r, \nu}$ from $\phi_{\mathcal{X}_{S} / S}^{r, \nu}$ via the base change by $\eta$ (see $\S 3$ for the details).

A key to the proof of Theorem 0.4 is the following construction: Put

$$
\begin{aligned}
& \Lambda^{r, \nu}\left(\mathcal{X}_{S} / S\right):=\operatorname{hom}_{\mathrm{MHS}}\left(\mathbb{Q}(0), H^{\nu}\left(S / \mathbb{C}, R^{2 r-\nu} f_{*} \mathbb{Q}(r)\right)\right), \\
& \Xi^{r, \nu}\left(\mathcal{X}_{S} / S\right):=\operatorname{Ext}_{\mathrm{MHS}}^{1}\left(\mathbb{Q}(0), H^{\nu-1}\left(S / \mathbb{C}, R^{2 r-\nu} f_{*} \mathbb{Q}(r)\right)\right),
\end{aligned}
$$

where $S / \mathbb{C}=S \times \overline{\mathbb{Q}} \mathbb{C}$, which we identify with its underlying complex manifold, and $H^{\bullet}\left(S / \mathbb{C}, R^{\bullet} f_{*} \mathbb{Q}(r)\right)$ denotes the cohomology of $S / \mathbb{C}$ with coefficients in a local system, which is endowed in a canonical way with a mixed Hodge structure by the theory of mixed Hodge modules ([MSa1]). (Alternatively, the reader can consult $[\mathrm{A}]$ for a different point of view.) Then one defines the natural maps

$$
\lambda_{\mathcal{X}_{S} / S}^{r, \nu}: F_{B}^{\nu} \mathrm{CH}^{r}\left(\mathcal{X}_{S} / S ; \mathbb{Q}\right) \rightarrow \Lambda^{r, \nu}\left(\mathcal{X}_{S} / S\right),
$$




$$
\epsilon_{\mathcal{X}_{S} / S}^{r, \nu}: \operatorname{ker}\left(\lambda_{\mathcal{X}_{S} / S}^{r, \nu}\right) \rightarrow \Xi^{r, \nu}\left(\mathcal{X}_{S} / S\right)
$$

Broadly speaking, the construction is given in terms of an extension class in the category of arithmetic mixed Hodge modules. We then construct the following commutative diagram under a suitable assumption on $\mathcal{X}_{S} / S$ (see Proposition 6.3)

$$
\begin{array}{rcc}
F_{B}^{\nu} \mathrm{CH}^{r}\left(\mathcal{X}_{S} / S ; \mathbb{Q}\right) & \stackrel{\phi_{\mathcal{X}_{S} / S}^{r, \nu}}{\longrightarrow} & \nabla J^{r, \nu}\left(\mathcal{X}_{S} / S\right) \\
\downarrow \lambda_{\mathcal{X}_{S} / S}^{r, \nu} & \stackrel{\Phi_{\mathcal{X}_{S} / S}^{r, \nu}}{\longrightarrow} & \nabla J^{r, \nu}\left(\mathcal{X}_{S} / S\right) \otimes_{\overline{\mathbb{Q}}} \mathbb{C} .
\end{array}
$$

Now the cycle classes in Theorem 0.4 are constructed in $\operatorname{ker}\left(\lambda_{\mathcal{X}_{S} / S}^{r, \nu}\right)$ and captured by $\epsilon_{\mathcal{X}_{S} / S}^{r, \nu}$.

The question now is whether the map $\Phi_{\mathcal{X}_{S} / S}^{r, \nu}$ in (0.5) always exists for $S$ affine. What we can say is that if one is willing to forgo the Hodge filtration by replacing the space of Mumford-Griffiths invariants $\nabla J^{r, \nu}\left(\mathcal{X}_{S} / S\right)$ in (0.5) by the coarser space of de Rham invariants $\nabla D R^{r, \nu}\left(\mathcal{X}_{S} / S\right)$ (see Definition 3.2), then for affine $S$, the diagram corresponding to $(0.5)$ does exist, and one subsequently obtains stronger results for cycles with trivial de Rham invariant (Theorem 7.2). We conjecture that for affine $S$, the map $\Lambda_{\text {alg }}^{r, \nu}\left(\mathcal{X}_{S} / S\right) \stackrel{\Phi_{\mathcal{X}_{S} / S}^{r, \nu}}{\longrightarrow} \nabla J^{r, \nu}\left(\mathcal{X}_{S} / S\right) \otimes_{\overline{\mathbb{Q}}} \mathbb{C}$ always exists, where $\Lambda_{\text {alg }}^{r, \nu}\left(\mathcal{X}_{S} / S\right)=\operatorname{Image}\left(\lambda_{\mathcal{X}_{S} / S}^{r, \nu}\right)$. (For a more precise statement, see Conjecture 6.4.) Roughly speaking, the discussion in the Appendix says that this conjecture is a consequence of the Hodge conjecture. If we put $S=\operatorname{Spec}(\mathbb{C})$ and let

$$
\begin{aligned}
\nabla J_{\text {alg }}^{r, \nu}(X) & :=\text { Image }\left\{G r_{F_{B}}^{\nu} \mathrm{CH}^{r}(X ; \mathbb{Q}) \stackrel{\phi_{X}^{r, \nu}}{\longrightarrow} \nabla J^{r, \nu}(X)\right\} \\
\nabla D R_{\text {alg }}^{r, \nu}(X) & :=\operatorname{Image}\left\{G r_{F_{B}}^{\nu} \mathrm{CH}^{r}(X ; \mathbb{Q}) \stackrel{\phi_{X, D R}^{r, \nu}}{\longrightarrow} \nabla D R^{r, \nu}(X)\right\},
\end{aligned}
$$

then a consequence of our aforementioned conjecture is that the natural map

$$
\nabla J_{\text {alg }}^{r, \nu}(X) \rightarrow \nabla D R_{\text {alg }}^{r, \nu}(X) .
$$

is an isomorphism. Put differently, and roughly speaking, the Hodge conjecture implies that to compute the Mumford-Griffiths invariant of an algebraic cycle, it is sufficient to compute its de Rham invariant. ${ }^{1}$

We are very grateful to Matt Kerr for meticulously reading parts of a preliminary version of this paper, and for providing useful comments; and in particular for sharing his ideas in [Ke2]. We are also grateful to V. Srinivas for pointing out his earlier work in [Sr]. Indeed the ideas presented in section 4 of this paper share with $[\mathrm{Sr}]$ a common methodology. Finally we want to thank the referees for impressing upon us the need to improve the presentation of our paper, by offering their numerous constructive comments.

\footnotetext{
${ }^{1}$ Since the submission of this paper, M. Saito shared with us his work in [MSa4], where now the conjectural assumptions, including Conjecture 6.4, are eliminated. Indeed as one of the referees pointed out, our Appendix seems already to have served as inspiration for [MSa4], and that our discussion is still valuable.
} 


\section{$\S 1$. Notation}

(i) All fields in this paper are considered as subfields of $\mathbb{C}$. For a field $k$, let $\mathcal{C}_{k}$ be the category of smooth projective varieties over $k$. We simply write $\mathcal{C}$ for $\mathcal{C}_{k}$ when $k=\mathbb{C}$.

(ii) Unless otherwise indicated, $X \in \mathcal{C}_{k}$ will denote a smooth projective variety of dimension $d$. (Periodically we will remind the reader that $\operatorname{dim} X=d$.) Then $\mathrm{CH}^{r}(X)$ is the Chow group of codimension $r$ algebraic cycles on $X$ modulo rational equivalence, and $\mathrm{CH}^{r}(X ; \mathbb{Q})=\mathrm{CH}^{r}(X) \otimes \mathbb{Q}$. Further, we put $A^{r}(X):=$ $\mathrm{CH}_{\text {alg }}^{r}(X ; \mathbb{Q}) \subset \mathrm{CH}^{r}(X ; \mathbb{Q})$ to be the subgroup of cycles algebraically equivalent to zero.

(iii) If $A \subset \mathbb{R}$ is a subring, we denote by $A(r),(2 \pi \sqrt{-1})^{r} A$ the corresponding Tate twist.

(iv) For $Y \in \mathcal{C}_{k}$, we write $Y_{\mathbb{C}}:=Y \times_{k} \mathbb{C}$. It is sometimes more convenient to use the notation $Y / \mathbb{C}$ for $Y_{\mathbb{C}}$, which we also identify with its underlying complex manifold.

(v) We fix a Weil cohomology theory for $\mathcal{C}_{k}$ :

$$
\mathcal{C}_{k} \rightarrow V e c ; X \rightarrow H^{\bullet}(X)
$$

where $V e c$ denotes the category of finite dimensional vector spaces over a fixed field of characteristic zero. Typical examples are given by

$$
X \rightarrow H^{\bullet}(X / \mathbb{C}, \mathbb{Q}) \quad\left(\text { singular cohomology) and } \quad X \rightarrow H_{\mathrm{DR}}^{\bullet}(X / k):=\mathbb{H}^{\bullet}\left(X, \Omega_{X / k}^{\bullet}\right) .\right.
$$

There are well-known standard conjectures with $\mathrm{Hg}(X) \Rightarrow B(X) \Rightarrow C(X)$ (where $\mathrm{Hg}(X)$ means the Hodge conjecture for $X)$. Let $X \in \mathcal{C}_{k}$ with $d=\operatorname{dim} X$ and let $L_{X} \in H^{2}(X)$ be a hyperplane class. Let $i \leq d$ be an integer. With regard to the hard Lefschetz isomorphism,

$$
L_{X}^{d-i}: H^{i}(X) \stackrel{\sim}{\longrightarrow} H^{2 d-i}(X),
$$

the hard Lefschetz conjecture $B(X)$ asserts that the inverse

$$
\left(L_{X}^{d-i}\right)^{-1}: H^{2 d-i}(X) \stackrel{\sim}{\rightarrow} H^{i}(X)
$$

is algebraic cycle induced. One consequence of $B(X)$ is a weaker conjecture $C(X)$ which asserts that the diagonal class $\left[\Delta_{X}\right] \in H^{2 d}(X \times X)$, has algebraic Künneth components:

$$
\left[\Delta_{X}\right]=\sum_{i+j=2 d}\left[\Delta_{X}(i, j)\right] \in \bigoplus_{i+j=2 d} H^{i}(X) \otimes H^{j}(X) \quad \text { with } \Delta_{X}(i, j) \in \mathrm{CH}^{d}(X \times X ; \mathbb{Q}) .
$$

The reader can consult [K1] for more details.

(vi) Let $f: X \rightarrow S$ be a smooth projective morphism of quasiprojective varieties over a base field $k$. We define $\mathrm{CH}^{r}(X / S ; \mathbb{Q})$ to be $\mathrm{CH}^{r}(X ; \mathbb{Q})$, and that " $/ S$ " will only affect how the filtration on $\mathrm{CH}^{r}(X / S ; \mathbb{Q})$ in Definition 2.8 is defined. 


\section{§2. Good Filtrations}

Fix a base field $k \subset \mathbb{C}$. Our goal is to work with a given filtration satisfying a number of good properties. More specifically, we require the following:

Definition 2.1. A filtration $F^{\bullet} C H^{\bullet}$ of Bloch-Beilinson type on $\mathcal{C}_{k}$ is given by the following data: For all $X \in \mathcal{C}_{k}$ and all $r$, there is a descending filtration

$$
C H^{r}(X ; \mathbb{Q})=F^{0} \supset F^{1} \supset \cdots \supset F^{\nu} \supset F^{\nu+1} \supset \cdots
$$

which satisfies the following

(i) $F^{0}=C H^{r}(X ; \mathbb{Q})$ and $F^{1}=C H_{\mathrm{hom}}^{r}(X ; \mathbb{Q})$.

(ii) $F^{\nu}$ is preserved under the action of correspondences: For $V, X \in \mathcal{C}_{k}$ and for $\Gamma \in C H^{q}(V \times X ; \mathbb{Q})$,

$$
\Gamma_{*}\left(F^{\nu} C H^{s}(V ; \mathbb{Q})\right) \subset F^{\nu} C H^{r}(X ; \mathbb{Q}),
$$

where $\Gamma_{*}: C H^{s}(V ; \mathbb{Q}) \rightarrow C H^{r}(X ; \mathbb{Q})$ with $s=r-q+\operatorname{dim}(V)$ is given by the formula

$$
\Gamma_{*}(\alpha)=\left(\pi_{X}\right)_{*}\left(\left(\pi_{V}\right)^{*}(\alpha) \bullet \Gamma\right) \quad \text { for } \alpha \in C H^{s}(V ; \mathbb{Q})
$$

where $\pi_{X}: V \times X \rightarrow X$ and $\pi_{V}: V \times X \rightarrow V$ are the projections.

(iii) The property (ii) implies that we have the induced map

$$
G r_{F}^{\nu} \Gamma_{*}: G r_{F}^{\nu} C H^{s}(V ; \mathbb{Q}) \rightarrow G r_{F}^{\nu} C H^{r}(X ; \mathbb{Q}) \text {. }
$$

Then $G r_{F}^{\nu} \Gamma_{*}$ is the zero map if so is $\varphi_{\Gamma}^{2 r-\nu}$ where $\varphi_{\Gamma}^{i}: H^{i-2(r-s)}(V) \rightarrow H^{i}(X)$ with $s=r-q+\operatorname{dim}(V)$ is given by the formula

$$
\varphi_{\Gamma}^{i}(\beta)=\left(\pi_{X}\right)_{*}\left(\left(\pi_{V}\right)^{*}(\beta) \cup[\Gamma]\right) \text { for } \beta \in H^{i-2(r-s)}(V),
$$

with $[\Gamma] \in H^{2 q}(V \times X)$, the cohomology class of $\Gamma$.

We point out the following consequences of Definition 2.1.

Proposition 2.2. Let $\mathrm{F}^{\bullet} \mathrm{CH}$ be a filtration of Bloch-Beilinson type on $\mathcal{C}_{k}$.

(i) $F^{\nu} C H^{\bullet}$ is preserved under push-forwards $f_{*}$ and pull-backs $f^{*}$ for a morphism $f: X \rightarrow Y$ in $\mathcal{C}_{k}$.

(ii) Let $X \in \mathcal{C}_{k}$ with $d=\operatorname{dim}(X)$. Assume $C(X)$ holds and let $\Delta_{X}(p, q) \in C H^{d}(X \times X ; \mathbb{Q})$ be as in $\S 1$ (v). Then

$$
\left.\Delta_{X}(2 d-2 r+\ell, 2 r-\ell)_{*}\right|_{G r_{F}^{\nu} \mathrm{CH}^{r}(X ; \mathbb{Q})}=\delta_{\ell, \nu} \cdot \text { Identity. }
$$

(iii) Under the same assumption as (ii),

$$
F^{\nu} C H^{r}(X ; \mathbb{Q}) \bullet F^{\mu} C H^{s}(X ; \mathbb{Q}) \subset F^{\nu+\mu} C H^{r+s}(X ; \mathbb{Q}),
$$

where $\bullet$ is the intersection product.

Proof. Parts (i) and (ii) follows immediately from the definition. Part (iii) is [Sa2], Theorem(0-2).

The following conjecture is due to Bloch and Beilinson: 
Conjecture 2.3. There exists a filtration $\mathrm{F}^{\bullet} \mathrm{CH}^{\bullet}$ of Bloch-Beilinson type satisfying:

$$
\bigcap_{\nu \geq 0} F^{\nu} C H^{r}(X ; \mathbb{Q})=0 \quad \text { for all } X \in \mathcal{C}_{k} \text { and all } r .
$$

In general one can put $D^{r}(X ; \mathbb{Q}):=\cap_{\nu \geq 0} F^{\nu} \mathrm{CH}^{r}(X ; \mathbb{Q})$ and work modulo " $D$-equivalence". We now introduce a specific filtration $F_{B}^{\bullet} \mathrm{CH}^{\bullet}$ of Bloch-Beilinson type, which is minimal among all filtrations of Bloch-Beilinson type, namely it satisfies $F_{B}^{\bullet} \mathrm{CH}^{\bullet} \subset F^{\bullet} \mathrm{CH}^{\bullet}$ for any filtration $F^{\bullet} \mathrm{CH}^{\bullet}$ of Bloch-Beilinson type. It is given in $[\mathrm{Sa} 2], \S 1$.

Definition 2.4. For $\nu \geq 0$ we define $F_{B}^{\nu} C H^{r}(X ; \mathbb{Q})$ for all $X \in \mathcal{C}_{k}$ and for all $r \geq 0$ inductively as follows:

(1) $F_{B}^{0} C H^{s}(V ; \mathbb{Q})=C H^{s}(V ; \mathbb{Q})$ for all $V \in \mathcal{C}_{k}$ and for all $s \geq 0$.

(2) Assume that we have defined $F_{B}^{\nu} C H^{s}(V ; \mathbb{Q})$ for all $V \in \mathcal{C}_{k}$ and for all $s \geq 0$. Then we define

$$
F_{B}^{\nu+1} C H^{r}(X ; \mathbb{Q})=\sum_{V, q, \Gamma} \operatorname{Image}\left(\Gamma_{*}: F_{B}^{\nu} C H^{r+d_{V}-q}(V ; \mathbb{Q}) \rightarrow C H^{r}(X ; \mathbb{Q})\right),
$$

where $V, q, \Gamma$ range over the following data:

(a) $V \in \mathcal{C}_{k}$ of dimension $d_{V}$,

(b) $r \leq q \leq r+d_{V}$,

(c) $\Gamma \in C H^{q}(V \times X ; \mathbb{Q})$ satisfying $\varphi_{\Gamma}^{2 r-\nu}=0$, where $\varphi_{\Gamma}^{2 r-\nu}: H^{2 s-\nu}(V) \rightarrow H^{2 r-\nu}(X)$ is as in Def. 2.1 (iii).

We have the following facts:

Proposition 2.5. ([Sa1] §4) Let $\rho_{X_{\mathbb{C}}}^{r}: C H_{\mathrm{hom}}^{r}(X ; \mathbb{Q}) \rightarrow J^{r}\left(X_{\mathbb{C}}\right) \otimes \mathbb{Q}$ be the Griffiths Abel-Jacobi map. Then

$$
F_{B}^{2} C H^{r}(X ; \mathbb{Q}) \subset \operatorname{ker}\left(\rho_{X_{\mathbb{C}}}^{r}\right) \quad \text { and } \quad F_{B}^{2} C H^{r}(X ; \mathbb{Q}) \cap A^{r}(X)=\operatorname{ker}\left(\rho_{X_{\mathbb{C}}}^{r}\right) \cap A^{r}(X) .
$$

Proposition 2.6. ([Sa2], Thm. (1-1)) Assume $C(X)$ (cf. $\S 1(v))$ for all $X \in \mathcal{C}_{k}$ and that Conjecture 2.3 holds. Then $\mathrm{F}_{B}^{\bullet} \mathrm{CH}$ is the only filtration of Bloch-Beilinson type.

Proposition 2.7. Let $X \subset \mathbb{P}^{N}$ be a smooth complete intersection of dimension d. For integers $r, \nu \geq 1$ we have:

$$
G r_{F_{B}}^{\nu} C H^{r}(X ; \mathbb{Q})=0 \quad \text { if } 2 r-\nu \neq d .
$$

Proof. By Lefschetz theory we have for $0 \leq i \leq 2 d$ :

$$
H^{i}(X)=\left\{\begin{array}{rl}
\mathbb{Q} \cdot[Y] & i \neq d, \text { even } \\
0 & i \neq d, \text { odd }
\end{array}\right.
$$

where for $i=2 m$, even, $j: Y \hookrightarrow X$ is the section by a general linear subspace of codimension $m$ and $[Y] \in H^{2 m}(X)$ denotes its cohomology class. Put $i=2 r-\nu$ and assume $i \geq 1$ and $i \neq d$. The diagonal $\Delta \subset X \times X$ induces

$$
\Delta_{*}: G r_{F_{B}}^{\nu} \mathrm{CH}^{r}(X ; \mathbb{Q}) \rightarrow G r_{F_{B}}^{\nu} \mathrm{CH}^{r}(X ; \mathbb{Q}) \quad \text { and } \quad \varphi_{\Delta}^{2 r-\nu}: H^{2 r-\nu}(X) \rightarrow H^{2 r-\nu}(X)
$$

and both maps are the identity. If $i$ is odd, then $\varphi_{\Delta}^{2 r-\nu}=0$ by $(*)$ and hence $\Delta_{*}=0$ by the definition of the filtration $F_{B}^{\bullet}$. If $i=2 m$, even, then $j_{*}: H^{0}(Y) \stackrel{\simeq}{\rightarrow} H^{2 m}(X)$ for $j: Y \hookrightarrow X$ as in $(*)$. Since $B(X)$ holds 
for smooth complete intersections, we can find $\Gamma \in \mathrm{CH}^{d_{Y}}(X \times Y ; \mathbb{Q})\left(d_{Y}=\operatorname{dim}(Y)=d-m\right)$ such that the induced map $\varphi_{\Gamma}^{0}: H^{2 m}(X) \rightarrow H^{0}(Y)$ is the inverse of the above map. Consider the induced maps

$$
\begin{gathered}
G r_{F_{B}}^{\nu} \mathrm{CH}^{r}(X ; \mathbb{Q}) \stackrel{\Gamma_{*}}{\longrightarrow} G r_{F_{B}}^{\nu} \mathrm{CH}^{r-m}(Y ; \mathbb{Q}) \stackrel{j_{*}}{\longrightarrow} G r_{F_{B}}^{\nu} \mathrm{CH}^{r}(X ; \mathbb{Q}), \\
H^{2 m}(X) \stackrel{\varphi_{\Gamma}^{0}}{\longrightarrow} H^{0}(Y) \stackrel{j_{*}}{\longrightarrow} H^{2 m}(X) .
\end{gathered}
$$

The composite of the maps in the second row is the identity and so is that in the first row by the definition of the filtration $F_{B}^{\bullet}$. Noting $r-m=\nu / 2<\nu,[\mathrm{Sa} 2]$ Cor.(3-2) implies $G_{F_{B}}^{\nu} \mathrm{CH}^{r-m}(Y ; \mathbb{Q})=0$ which needs the fact that $B(Y)$ holds. This proves the desired assertion.

There is a variational (or relative) version of the aforementioned filtration. The definition is completely analogous to that given in [Sa3], Definition (2-1). As a base we fix a localization $S$ of a smooth quasiprojective variety $S$ over a field and let $\mathcal{C}_{S}$ be the category of $f: X \rightarrow S$, smooth projective morphisms. ${ }^{2}$ For $f: X \rightarrow S$ in $\mathcal{C}_{S}$, let $\Omega_{X / S}^{\bullet}$ be the de Rham complex of (Zariski) sheaves of relative differential forms of $X$ over $S$. We define the de Rham cohomology sheaf:

$$
\mathcal{H}_{\mathrm{DR}}^{i}(X / S):=\mathbb{R}^{i} f_{*}\left(\Omega_{X / S}^{\bullet}\right),
$$

which is the Zarisiki sheaf of $\mathcal{O}_{S}$-modules.

There is a direct generalization of Definition 2.4 to the relative case.

Definition 2.8. For all $r, \nu \geq 0$ and for all $X \in \mathcal{C}_{S}$, we define $F_{B}^{\nu} C H^{r}(X / S ; \mathbb{Q}) \subset C H^{r}(X ; \mathbb{Q})$ in the following inductive way:

(1) $F_{B}^{0} C H^{s}(V / S ; \mathbb{Q})=C H^{s}(V ; \mathbb{Q})$ for all $V \in \mathcal{C}_{S}$ and for all $s \geq 0$.

(2) Assume that we have defined $F_{B}^{\nu} C H^{s}(V / S ; \mathbb{Q})$ for all $V \in \mathcal{C}_{S}$ and for all $s \geq 0$. Then we define

$$
F_{B}^{\nu+1} C H^{r}(X / S ; \mathbb{Q})=\sum_{V, q, \Gamma} \operatorname{Image}\left(\Gamma_{*}: F_{B}^{\nu} C H^{r+d_{V}-q}(V / S ; \mathbb{Q}) \rightarrow C H^{r}(X ; \mathbb{Q})\right),
$$

where $V, q, \Gamma$ range over the following data:

(a) $V \in \mathcal{C}_{S}$ of relative dimension $d_{V}$,

(b) $r \leq q \leq r+d_{V}$,

(c) $\Gamma \in C H^{q}(V \times X ; \mathbb{Q})$ satisfying the condition $\varphi_{\Gamma, D R / S}^{2 r-\nu}=0$, where

$$
\varphi_{\Gamma, D R / S}^{i}: \mathcal{H}_{\mathrm{DR}}^{i-2(r-s)}(V / S) \rightarrow \mathcal{H}_{\mathrm{DR}}^{i}(X / S) \quad\left(s=r-q+d_{V}\right),
$$

is the homomorphism of $\mathcal{O}_{S}$-modules, which is given by the same formula as in Definition 2.1 (iii), though using $[\Gamma] \in H^{0}\left(S, \mathcal{H}_{\mathrm{DR}}^{2 q}(V \times X / S)\right)$, the cohomology class of $\Gamma$ in the de Rham cohomology.

We note the following functoriality of the above filtration: Let $\tau: T \rightarrow S$ be a morphism of schemes which are localizations of smooth quasiprojective varieties over a field. For $f: X \rightarrow S$ in $\mathcal{C}_{S}$ let $X_{T}=X \times_{S} T \in \mathcal{C}_{T}$ be the base change via $\tau$. Then, we have $\tau^{*}\left(F_{B}^{\nu} \mathrm{CH}^{r}(X / S ; \mathbb{Q})\right) \subset F_{B}^{\nu} \mathrm{CH}^{r}\left(X_{T} / T ; \mathbb{Q}\right)$ under the pull-back $\tau^{*}: \mathrm{CH}^{r}(X ; \mathbb{Q}) \rightarrow \mathrm{CH}^{r}\left(X_{T} ; \mathbb{Q}\right)$. If $\tau: T=\operatorname{Spec}(k) \rightarrow S$ is the generic point of $S$, this gives the compatibility of absolute and relative versions of the filtrations on Chow groups in Definition 2.4 and 2.8.

\footnotetext{
${ }^{2}$ We remind the reader that a smooth morphism is a submersion at every point.
} 


\section{§3. Arithmetic de Rham cohomology}

Many of the ideas presented here in this section are inspired by the lectures of M. Green [Gr]. Fix a base field $k \subset \mathbb{C}$. Let $\mathcal{S}_{k}$ be the category of the affine integral schemes which are localizations of smooth schemes over $k$. For example, for a finitely generated separable extension of fields $K / k, \operatorname{Spec}(K)$ is an object of $\mathcal{S}_{k}$.

3.1. Arithmetic Gauss-Manin connection. We recall the definition of the arithmetic Gauss-Manin connection. Take $S=\operatorname{Spec}(R) \in \mathcal{S}_{k}$ and let $f: X \rightarrow S$ be smooth projective. Let $\Omega_{X_{S} / k}^{1}$ (resp. $\Omega_{X / S}^{1}$ ) be the Zariski sheaf of relative differential forms of $X$ over $k$ (resp. $S$ ) and put $\Omega_{X_{S} / k}^{p}=\stackrel{p}{\wedge} \Omega_{X_{S} / k}^{1}$ (resp. $\left.\Omega_{X / S}^{p}=\wedge_{\wedge}^{p} \Omega_{X / S}^{1}\right)$. Note that $\Omega_{X_{S} / k}^{\bullet}$ is a complex under $d$. We define the de Rham cohomology groups:

$$
\begin{gathered}
H_{\mathrm{DR}}^{i}\left(X_{S} / k\right):=\mathbb{H}^{i}\left(X_{Z a r}, \Omega_{X_{S} / k}^{\bullet}\right)=H^{i}\left(S_{Z a r}, \mathbb{R} f_{*} \Omega_{X_{S} / k}^{\bullet}\right), \\
H_{\mathrm{DR}}^{i}(X / S):=\mathbb{H}^{i}\left(X_{Z a r}, \Omega_{X / S}^{\bullet}\right)=H^{i}\left(S_{Z a r}, \mathbb{R} f_{*} \Omega_{X / S}^{\bullet}\right) .
\end{gathered}
$$

Put

$$
\text { Filt }^{m} \Omega_{X_{S} / k}^{p}:=\operatorname{Im}\left(\Omega_{R / k}^{m} \otimes \Omega_{X_{S} / k}^{p-m} \rightarrow \Omega_{X_{S} / k}^{p}\right) .
$$

Then:

$$
\mathrm{Gr}^{m} \Omega_{X_{S} / k}^{p} \simeq \Omega_{R / k}^{m} \otimes \Omega_{X / S}^{p-m}
$$

Moreover:

$$
\begin{gathered}
0 \rightarrow \frac{\operatorname{Filt}^{1} \Omega_{X_{S} / k}^{\bullet}}{\operatorname{Filt}^{2} \Omega_{X_{S} / k}^{\bullet}} \rightarrow \frac{\Omega_{X_{S} / k}^{\bullet}}{\operatorname{Filt}^{2} \Omega_{X_{S} / k}^{\bullet}} \rightarrow \frac{\Omega_{X_{S} / k}^{\bullet}}{\operatorname{Filt}^{1} \Omega_{X_{S} / k}^{\bullet}} \rightarrow 0 \\
|2 \quad| \mid \quad \\
0 \rightarrow \Omega_{R / k}^{1} \otimes \Omega_{X / S}^{\bullet}[-1] \rightarrow \frac{\Omega_{X_{S} / k}^{\bullet}}{\operatorname{Filt}^{2} \Omega_{X / S}^{\bullet}} \rightarrow \Omega_{X / S}^{\bullet} \rightarrow 0
\end{gathered}
$$

Taking hypercohomology, we get a natural connecting map:

$$
\nabla:=\nabla_{X_{S} / k}: H_{\mathrm{DR}}^{i}(X / S) \rightarrow \Omega_{R / k}^{1} \otimes H_{\mathrm{DR}}^{i}(X / S),
$$

called the arithmetic Gauss-Manin connection. By imposing Leibniz' rule, viz.,

$$
\nabla(\omega \otimes e)=d \omega \otimes e+(-1)^{m} \omega \otimes \nabla e
$$

one extends $\nabla$ to:

$$
\nabla: \Omega_{R / k}^{m} \otimes H_{\mathrm{DR}}^{i}(X / S) \rightarrow \Omega_{R / k}^{m+1} \otimes H_{\mathrm{DR}}^{i}(X / S) .
$$

It satisfies the following properties, which are consequences of the fact that $\nabla$ is identified with $d_{1}$ of the spectral sequences (3.1.1) and (3.1.2) below by [KO].

(i) $\nabla^{2}=0$ (viz., flat connection),

(ii) (Griffiths transversality)

$$
\nabla\left(F^{p} H_{\mathrm{DR}}^{i}(X / S)\right) \subset \Omega_{R / k}^{1} \otimes F^{p-1} H_{\mathrm{DR}}^{i}(X / S),
$$

where $F^{p} H_{\mathrm{DR}}^{\bullet}(X / S):=\mathbb{H}^{\bullet}\left(\Omega_{X / S}^{\bullet \geq p}\right) \subset \mathbb{H}^{\bullet}\left(\Omega_{X / S}^{\bullet}\right)$ is the usual Hodge filtration. 
Note that $\Omega_{X_{S} / k}^{\bullet}$ is a filtered complex using Filt $^{m}$. The corresponding spectral sequence is:

$$
E_{1}^{p, q}=\Omega_{R / k}^{p} \otimes H_{\mathrm{DR}}^{q}(X / S) \Rightarrow H_{\mathrm{DR}}^{p+q}\left(X_{S} / k\right),
$$

with $d_{1}=\nabla_{X_{S} / k}([\mathrm{KO}])$. This is really the Leray spectral sequence, which by Deligne ([De3]), is known to degenerate $E_{2}$. Likewise, the analogous Leray spectral sequence

$$
E_{1}^{p, q}=\Omega_{R / k}^{p} \otimes F^{r-p} H_{\mathrm{DR}}^{q}(X / S) \Rightarrow \mathbb{H}^{2 r}\left(\Omega_{X_{S} / k}^{\bullet \geq r}\right)
$$

degenerates at $E_{2}$. We denote the corresponding Leray filtrations by:

$$
F_{L}^{\nu} \mathbb{H}^{\bullet}\left(\Omega_{X_{S} / k}^{\bullet}\right) \subset \mathbb{H}^{\bullet}\left(\Omega_{X_{S} / k}^{\bullet}\right), \quad F_{L}^{\nu} \mathbb{H}^{\bullet}\left(\Omega_{X_{S} / k}^{\bullet \geq r}\right) \subset \mathbb{H}^{\bullet}\left(\Omega_{X_{S} / k}^{\bullet \geq r}\right)
$$

Definition 3.2. (i) We put $\nabla J^{r, \nu}(X / S)=G r_{F_{L}}^{\nu} \mathbb{H}^{2 r}\left(\Omega_{X_{S} / k}^{\bullet \geq r}\right)$, called the space of Mumford-Griffiths invariants of $X / S$. It is given by the cohomology of:

$$
\Omega_{R / k}^{\nu-1} \otimes F^{r-\nu+1} H_{\mathrm{DR}}^{2 r-\nu}(X / S) \stackrel{\nabla}{\longrightarrow} \Omega_{R / k}^{\nu} \otimes F^{r-\nu} H_{\mathrm{DR}}^{2 r-\nu}(X / S) \stackrel{\nabla}{\longrightarrow} \Omega_{R / k}^{\nu+1} \otimes F^{r-\nu-1} H_{\mathrm{DR}}^{2 r-\nu}(X / S) .
$$

(ii) We put $\nabla D R^{r, \nu}(X / S)=G r_{F_{L}}^{\nu} \mathbb{H}^{2 r}\left(\Omega_{X_{S} / k}^{\bullet}\right)$, called the space of de Rham invariants. It is given by the cohomology of:

$$
\Omega_{R / k}^{\nu-1} \otimes H_{\mathrm{DR}}^{2 r-\nu}(X / S) \stackrel{\nabla}{\longrightarrow} \Omega_{R / k}^{\nu} \otimes H_{\mathrm{DR}}^{2 r-\nu}(X / S) \stackrel{\nabla}{\longrightarrow} \Omega_{R / k}^{\nu+1} \otimes H_{\mathrm{DR}}^{2 r-\nu}(X / S) .
$$

(iii) In case $S=\operatorname{Spec}(\mathbb{C})$ we simply write $\nabla J^{r, \nu}(X)=\nabla J^{r, \nu}(X / S)$ and $\nabla D R^{r, \nu}(X)=\nabla D R^{r, \nu}(X / S)$.

Note that there is a natural map $\nabla J^{r, \nu}(X / S) \rightarrow \nabla D R^{r, \nu}(X / S)$, where one forgets the Hodge filtration. 3.3. Arithmetic cycle class map. Let $f: X \rightarrow S=\operatorname{Spec}(R)$ be as before. Let

$$
\mathcal{K}_{r, X}^{M}=\mathcal{O}_{X}^{\times} \otimes \cdots \otimes \mathcal{O}_{X}^{\times} /\left\langle\tau_{1} \otimes \cdots \otimes \tau_{i} \otimes \cdots \otimes \tau_{j} \otimes \cdots \otimes \tau_{r} \mid \tau_{i}+\tau_{j}=1, i \neq j\right\rangle,
$$

be the Milnor $K$-sheaf of $X$, and put

$$
\underline{\mathcal{K}}_{r, X}^{M}=\operatorname{Image}\left(\mathcal{K}_{r, X}^{M} \rightarrow K_{r}^{M}(k(X))\right) .
$$

where $k(X)$ is the function field of $X$. By the results of Gabber (or Müller-Stach, Elbaz-Vincent, see [E-M])

$$
\mathrm{CH}^{r}(X) \simeq H_{\mathrm{Zar}}^{r}\left(X, \underline{\mathcal{K}}_{r, X}^{M}\right)=\mathbb{H}^{r}\left(\underline{\mathcal{K}}_{r, X}^{M} \rightarrow 0 \rightarrow 0 \rightarrow \cdots\right)
$$

By torsion considerations (Suslin), the natural map $\mathcal{K}_{r, X}^{M} \rightarrow \Omega_{X_{S} / k}^{\bullet \geq r}[r]$ given by

$$
\left\{f_{1}, \ldots, f_{r}\right\} \mapsto \bigwedge_{j} d \log f_{j}, f_{j} \in \mathcal{O}_{X}^{\times}
$$

factors through a morphism of complexes

$$
\left(\underline{\mathcal{K}}_{r, X}^{M} \rightarrow 0 \rightarrow 0 \rightarrow \cdots\right) \rightarrow \Omega_{X_{S} / k}^{\bullet r}[r]
$$


$\left(\underline{\mathcal{K}}_{r, X}^{M}\right.$ and $\Omega_{X_{S} / k}^{r}$ are in degree 0$)$ and hence determines a morphism

$$
c_{X_{S} / k}^{r}: \mathrm{CH}^{r}(X ; \mathbb{Q}) \rightarrow \mathbb{H}^{2 r}\left(\Omega_{X_{S} / k}^{\bullet \geq r}\right),
$$

called the arithmetic cycle class map. The same construction also appears in [Gr], based on the discussion on p. 68 in [E-P] and Théorèm 5 in [So]. (The reader can also consult the treatment by El-Zein [EZ].) We will also use

$$
c_{X_{S} / k, D R}^{r}: \mathrm{CH}^{r}(X ; \mathbb{Q}) \rightarrow \mathbb{H}^{2 r}\left(\Omega_{X_{S} / k}^{\bullet}\right),
$$

which is the composite of $c_{X_{S} / k}^{r}$ and $\mathbb{H}^{2 r}\left(\Omega_{X_{S} / k}^{\bullet \geq r}\right) \rightarrow \mathbb{H}^{2 r}\left(\Omega_{X_{S} / k}^{\bullet}\right)$, the natural map. These maps satisfy functoriality with respect to a base change $\pi: S^{\prime} \rightarrow S$ in $\mathcal{S}_{k}$, namely we have the commutative diagrams $\left(X^{\prime}=X \times{ }_{S} S^{\prime}\right)$ :

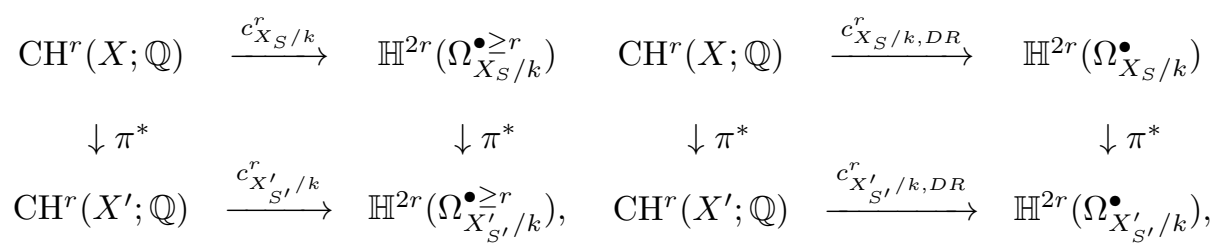

Definition 3.5. We introduce filtrations of Leray type on $C H^{r}(X / S)$ as follows:

$$
\begin{gathered}
F_{\psi / k}^{\nu} C H^{r}(X / S)=\left(c_{X_{S} / k}^{r}\right)^{-1}\left(F_{L}^{\nu} \mathbb{H}^{2 r}\left(\Omega_{X_{S} / k}^{\bullet \geq r}\right)\right), \\
F_{\mathrm{DR} / k}^{\nu} C H^{r}(X / S)=\left(c_{X_{S} / k, D R}^{r}\right)^{-1}\left(F_{L}^{\nu} \mathbb{H}^{2 r}\left(\Omega_{X_{S} / k}^{\bullet}\right)\right) .
\end{gathered}
$$

By definition we have $F_{\psi / k}^{\nu} \mathrm{CH}^{r}(X / S) \subset F_{\mathrm{DR} / k}^{\nu} \mathrm{CH}^{r}(X / S)$.

Lemma 3.6. Let $\pi: S^{\prime} \rightarrow S$ be a morphism in $\mathcal{S}_{k}$ and put $X^{\prime}=X \times{ }_{S} S^{\prime}$. We have

$$
\pi^{*}\left(F_{\psi / k}^{\nu} C H^{r}(X / S)\right) \subset F_{\psi / k}^{\nu} C H^{r}\left(X^{\prime} / S^{\prime}\right), \quad \pi^{*}\left(F_{\mathrm{DR} / k}^{\nu} C H^{r}(X / S)\right) \subset F_{\mathrm{DR} / k}^{\nu} C H^{r}\left(X^{\prime} / S^{\prime}\right) .
$$

If $\pi$ is finite etale, then

$$
\pi_{*}\left(F_{\psi / k}^{\nu} C H^{r}\left(X^{\prime} / S^{\prime}\right)\right) \subset F_{\psi / k}^{\nu} C H^{r}(X / S), \quad \pi_{*}\left(F_{\mathrm{DR} / k}^{\nu} C H^{r}\left(X^{\prime} / S^{\prime}\right)\right) \subset F_{\mathrm{DR} / k}^{\nu} C H^{r}(X / S) .
$$

Proof. This follows from the corresponding functoriality for (3.1.1) and (3.1.2).

Now recall $\left\{F_{B}^{\nu} \mathrm{CH}^{r}(X / S ; \mathbb{Q})\right\}_{\nu \geq 0}$ as introduced earlier in Definition 2.4 of $\S 2$.

Proposition 3.7. For all $\nu \geq 0, F_{B}^{\nu} C H^{r}(X / S ; \mathbb{Q}) \subset F_{\psi / k}^{\nu} C H^{r}(X / S)$. Hence there exists a natural map

$$
\phi_{X / S}^{r, \nu}: F_{B}^{\nu} C H^{r}(X / S ; \mathbb{Q}) \rightarrow \nabla J^{r, \nu}(X / S)
$$

satisfying $\phi_{X / S}^{r, \nu}\left(F_{B}^{\nu+1} C H^{r}(X / S ; \mathbb{Q})\right)=0$. It is functorial for morphisms $Y \rightarrow X$ of projective smooth schemes over $S$, also for base change $S^{\prime} \rightarrow S$. 
Proof. This is shown by the same argument as [Sa3], Prop.(2-1): Let $\Gamma \in \mathrm{CH}^{q}(V \times X ; \mathbb{Q})$ be as Definition 2.8 (c). It induces a commutative diagram:

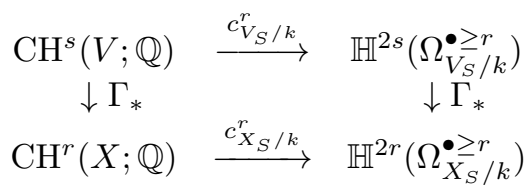

where the vertical maps respect $F_{B}^{\nu}$ on Chow groups and $F_{L}^{\nu}$ introduced in (3.1.3). The induced map

$$
G r_{F_{L}}^{\nu} \Gamma_{*}: G r_{F_{L}}^{\nu} \mathbb{H}^{2 s}\left(\Omega_{V_{S} / k}^{\bullet \geq r}\right) \rightarrow G r_{F_{L}}^{\nu} \mathbb{H}^{2 r}\left(\Omega_{X_{S} / k}^{\bullet \geq r}\right)
$$

is then identified with the map induced by $\Gamma_{*}: H_{\mathrm{DR}}^{2 s-\nu}(V / S) \rightarrow H_{\mathrm{DR}}^{2 r-\nu}(X / S)$ via the identification of the above spaces with the cohomology of the complex in Definition 3.2 (i). The proposition follows by the induction on $\nu$.

We let

$$
\phi_{X / S, D R}^{r, \nu}: F_{B}^{\nu} \mathrm{CH}^{r}(X / S ; \mathbb{Q}) \rightarrow \nabla D R^{r, \nu}(X / S),
$$

denote the composite of $\phi_{X / S}^{r, \nu}$ and $\nabla J^{r, \nu}(X / S) \rightarrow \nabla D R^{r, \nu}(X / S)$, the natural map.

In the case $S=\operatorname{Spec}(\mathbb{C})$ and $X \in \mathcal{C}$ we get the maps

$$
\begin{gathered}
\phi_{X}^{r, \nu}:=\phi_{X / \mathbb{C}}^{r, \nu}: F_{B}^{\nu} \mathrm{CH}^{r}(X ; \mathbb{Q}) \rightarrow \nabla J^{r, \nu}(X) . \\
\phi_{X, D R}^{r, \nu}:=\phi_{X / \mathbb{C}}^{r, \nu}: F_{B}^{\nu} \mathrm{CH}^{r}(X ; \mathbb{Q}) \rightarrow \nabla D R^{r, \nu}(X) .
\end{gathered}
$$

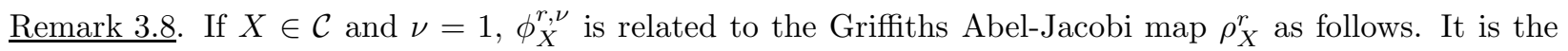
Griffiths construction of infinitesimal invariant of normal functions. We recall

$$
J^{r}(X)=H^{2 r-1}\left(X_{a n}, \mathbb{C}\right) /\left(F^{r} H^{2 r-1}\left(X_{a n}, \mathbb{C}\right)+H^{2 r-1}\left(X_{a n}, \mathbb{Z}(r)\right)\right) .
$$

We have the comparison isomorphism $H^{q}\left(X_{a n}, \mathbb{C}\right) \simeq H_{\mathrm{DR}}^{q}(X / \mathbb{C})$ preserving the Hodge filtrations and the arithmetic Gauss-Manin connection $\nabla$ annihilates the image of the subspace $H^{q}\left(X_{a n}, \mathbb{Q}(r)\right) \subset H^{q}\left(X_{a n}, \mathbb{C}\right)$ for every $r$. Hence $\nabla$ induces

$$
\tau: J^{r}(X) \rightarrow \nabla J^{r, 1}(X),
$$

and one can check that $\phi_{X}^{r, 1}=\tau \circ \rho_{X}^{r}$. 


\section{$\S 4$. Capturing nontrivial Classes via $\phi_{X}^{r, \nu}$}

Let $\mathcal{F}$ denote the set of the subfields $\mathbb{Q} \subset k \subset \mathbb{C}$ whose transcendental degree over $\mathbb{Q}$ is at most countable (bear in mind that $\mathbb{C}$ has uncountable transcendence degree over $\mathbb{Q}$ ). We want to prove infinite dimensionality statements about the image of $\phi_{X}^{r, \nu}$ (from (3.7.2)) under certain conditions on $X$. In this section, we will actually prove infinite dimensionality results for the de Rham invariants, which immediately imply the same for the Mumford-Griffiths invariants.

First, we need to introduce some integral invariants. We pick a base field $k \in \mathcal{F}$.

Definition 4.1. Let $X \in \mathcal{C}$ be a projective smooth variety of dimension $d$ over $\mathbb{C}$. Fix an integer $\nu>1, \Xi$ a subset of $A^{r}(X)$ (cf. $\left.\S 1(i i)\right)$. We define $r k_{\mathrm{DR} / k}^{(\nu)}(\Xi)$ to be the least of the integers $\mu$ for which the following holds: There exists $\left\{f_{i}: Y_{o i} \times_{k} \mathbb{C} \rightarrow X\right\}_{i \in \mathbb{N}}$, a countable set of morphisms in $\mathcal{C}$ with $Y_{\text {oi }} \in \mathcal{C}_{k}$ such that $\operatorname{dim} Y_{o i} \leq d-r+\mu$ for all $i$ and

$$
\Xi \subset \operatorname{Image}\left\{\bigoplus_{i \in \mathbb{N}} C H_{d-r}\left(Y_{i}\right) \stackrel{f_{*}}{\longrightarrow} C H^{r}(X)\right\}+F_{\mathrm{DR} / k}^{\nu+1} C H^{r}(X / \mathbb{C}),
$$

where $f_{*}$ is induced by $f_{i}$ for $i \in \mathbb{N}, F_{\mathrm{DR} / k}^{\nu+1} C H^{r}(X / \mathbb{C})$ is as in Definition 3.5 with $S=S p e c(\mathbb{C})$, and $Y_{i}=Y_{o i} \times_{k} \mathbb{C}$. We also define

$$
r k_{\mathrm{DR}}^{(\nu)}(\Xi)=\min \left\{r k_{\mathrm{DR} / k}^{(\nu)}(\Xi) \mid k \in \mathcal{F}\right\} .
$$

Note that $r k_{\mathrm{DR} / k}^{(\nu)}(\Xi) \geq r k_{\mathrm{DR} / k^{\prime}}^{(\nu)}(\Xi)$ if $k, k^{\prime} \in \mathcal{F}$ and $k \subset k^{\prime}$, and that

$$
0 \leq r k_{\mathrm{DR}}^{(\nu)}(\Xi) \leq r k_{\mathrm{DR} / k}^{(\nu)}(\Xi) \leq r .
$$

Our main result is the following:

Theorem 4.2. Let $W, X \in \mathcal{C}$ with $\operatorname{dim} X=d$ and $\Gamma \in C H^{r}(W \times X)$ be given. Write

$$
r k_{\mathrm{DR}}^{(\nu)}(\Gamma):=r k_{\mathrm{DR}}^{(\nu)}\left(\Gamma_{*}\left(A_{0}(W)\right)\right),
$$

where $\Gamma_{*}: A_{0}(W) \rightarrow A^{r}(X)$ is induced by $\Gamma$. Then we have the implication

$$
r k_{\mathrm{DR}}^{(\nu)}(\Gamma) \leq \nu-1 \Rightarrow \operatorname{Image}\left(\varphi_{\Gamma}^{\nu}\right) \subset F^{1} H_{\mathrm{DR}}^{\nu}(W / \mathbb{C}),
$$

with $\varphi_{\Gamma}^{\nu}: H_{\mathrm{DR}}^{2(d-r)+\nu}(X / \mathbb{C}) \rightarrow H_{\mathrm{DR}}^{\nu}(W / \mathbb{C})$ as in Def. 2.1 (iii), and where $F^{\bullet}$ is the Hodge filtration.

The proof of this theorem proceeds in the same way as [Sa1], $\S 7$ and $\S 8$. It hinges on the following key lemma, which is an arithmetic counterpart of [Sa1], Theorem 7.1:

Lemma 4.3. Let $W, X \in \mathcal{C}_{k}, \Gamma \in C H^{r}(W \times X)$ be given with $d=\operatorname{dim} X$. Introduce $m=d-r$ in Definition 4.1. Let $\eta$ be the generic point of $W$ and put $K=k(\eta)$ and $X_{K}=X \times_{k} K$. Assume given a k-rational point $a \in W$ and put

$$
\xi:=\Gamma \bullet(\eta \times X)-\Gamma \bullet(a \times X) \in C H^{r}\left(X_{K}\right) .
$$

where - denotes the intersection product. Suppose that there exists $\left\{f_{i}: Y_{i} \rightarrow X\right\}_{i \in \mathbb{N}}$, a countable set of morphisms in $\mathcal{C}_{k}$ such that $\operatorname{dim} Y_{i} \leq m+\nu-1$ for all $i$ and

$$
\xi \in \operatorname{Image}\left(\bigoplus_{i \in \mathbb{N}} C H_{m}\left(Y_{i K} ; \mathbb{Q}\right) \stackrel{f_{*}}{\longrightarrow} C H^{r}\left(X_{K}, \mathbb{Q}\right)\right)+F_{\mathrm{DR} / k}^{\nu+1} C H^{r}\left(X_{K} / K\right), \quad\left(Y_{i K}=Y_{i} \times_{k} K\right)
$$


where $f_{*}$ is induced by $f_{i}$ for $i \in \mathbb{N}$ and $F_{\mathrm{DR} / k}^{\nu+1} C H^{r}\left(X_{K} / K\right)$ is defined as in Definition 3.5 for $S=\operatorname{Spec}(K)$. Then we have

$$
\operatorname{Image}\left(\varphi_{\Gamma}^{\nu}\right) \subset F^{1} H_{\mathrm{DR}}^{\nu}(W / k),
$$

where $\varphi_{\Gamma}^{\nu}: H_{\mathrm{DR}}^{2(d-r)+\nu}(X / k) \rightarrow H_{\mathrm{DR}}^{\nu}(W / k)$ is as in Def. 2.1 (iii).

Proof. The idea of the proof originates from Salberger's duality interpretation of Mumford's theorem (cf. [Bl] $\S 1$ Appendix): Writing for $K=k(\eta)$ and defining

$$
H_{\mathrm{DR}}^{p}(K / k)=\lim _{U \vec{\subset} W} H_{\mathrm{DR}}^{p}(U),
$$

with $U$ ranging over all nonempty Zariski open subsets of $W$, put

$$
\Phi_{\mathrm{DR}}^{\nu}(W)=\frac{H_{\mathrm{DR}}^{\nu}(W / k)}{\operatorname{ker}\left(H_{\mathrm{DR}}^{\nu}(W / k) \rightarrow H_{\mathrm{DR}}^{\nu}(K / k)\right)} .
$$

The idea is to use a pairing defined for any $X \in \mathcal{C}_{k}$ :

$$
\langle,\rangle_{W, X}^{(\nu)}: \mathrm{CH}^{r}\left(X_{K} ; \mathbb{Q}\right) \otimes H_{\mathrm{DR}}^{2 m+\nu}(X / k) \rightarrow \Phi_{\mathrm{DR}}^{\nu}(W),
$$

which satisfies the following properties:

(i) Let $\xi \in \mathrm{CH}^{r}\left(X_{K} ; \mathbb{Q}\right)$ be as in Lemma 4.3. Then

$$
\langle\xi, \beta\rangle_{W, X}^{(\nu)}=0 \text { for any } \beta \in H_{\mathrm{DR}}^{2 m+\nu}(X / k) \Rightarrow \operatorname{Image}\left(\varphi_{\Gamma}^{\nu}\right) \subset F^{1} H_{\mathrm{DR}}^{\nu}(W / k) .
$$

(ii) $\langle,\rangle_{W, X}^{(\nu)}$ annihilates $F_{\mathrm{DR} / k}^{\nu+1} \mathrm{CH}^{r}\left(X_{K} / K\right)$ placed on the left-hand side.

(iii) If $f: Y \rightarrow X$ is a morphism in $\mathcal{C}_{k}$, there is a commutative diagram

$$
\begin{array}{ccccccc}
\langle,\rangle_{W, Y}^{(\nu)}: & \mathrm{CH}^{r}\left(Y_{K}\right) & \otimes & H_{\mathrm{DR}}^{2 m+\nu}(Y / k) & \rightarrow & \Phi_{\mathrm{DR}}^{\nu}(W) \\
& \downarrow f_{*} & & \uparrow f^{*} & & \| \\
\langle,\rangle_{W, X}^{(\nu)} & : & \mathrm{CH}^{r}\left(X_{K}\right) \otimes & H_{\mathrm{DR}}^{2 m+\nu}(X / k) & \rightarrow & \Phi_{\mathrm{DR}}^{\nu}(W)
\end{array}
$$

(iv) Recall $m=d-r$. Then $\langle,\rangle_{W, Y}^{(\nu)}$ is trivial if $\operatorname{dim}(Y) \leq m+\nu-1$.

Lemma 4.3 follows immediately from this. To construct the pairing, we note the decomposition

$$
\mathbb{H}^{2 r}\left(X_{K}, \Omega_{X_{K}(K) / k}\right)=\bigoplus_{p+q=2 r} H_{\mathrm{DR}}^{p}(K / k) \otimes_{K} H_{\mathrm{DR}}^{q}\left(X_{K} / K\right)=\bigoplus_{p+q=2 r} H_{\mathrm{DR}}^{p}(K / k) \otimes_{k} H_{\mathrm{DR}}^{q}(X / k),
$$

which is a consequence of the degeneration at $E_{1}$ of the Leray spectral sequence

$$
E_{1}^{p, q}=\Omega_{K / k}^{p} \otimes_{K} H_{\mathrm{DR}}^{q}\left(X_{K} / K\right) \Rightarrow \mathbb{H}^{p+q}\left(X_{K}, \Omega_{X_{K}(K) / k}^{\bullet}\right)
$$

following from the product structure $W \times X$ over $k$, and de Rham base change (alternatively, one may apply the Künneth formula to $\Omega_{X_{K}(K) / k} \simeq \Omega_{K / k}^{\bullet} \otimes_{k} \Omega_{X / k}^{\bullet}$, where the differential $d$ on the left hand side is induced by $d_{K / k} \otimes 1+(-1)^{\bullet}\left(1 \otimes d_{X / k}\right)$ on the right hand side). Thus from the product structure $W \times X$ over $k$, we have the Leray filtration

$$
F_{L}^{\nu} \mathbb{H}^{2 r}\left(X_{K}, \Omega_{X_{K}(K) / k}^{\bullet}\right)=\bigoplus_{p \geq \nu, p+q=2 r} H_{\mathrm{DR}}^{p}(K / k) \otimes_{k} H_{\mathrm{DR}}^{q}(X / k)
$$


Then by composing $c_{X_{K}(K) / k, D R}^{r}$ for $X_{K}$ in 3.3 .2 with the projection

$$
\mathbb{H}^{2 r}\left(X_{K}, \Omega_{X_{K}(K) / k}\right) \rightarrow H_{\mathrm{DR}}^{\nu}(K / k) \otimes_{k} H_{\mathrm{DR}}^{2 r-\nu}(X / k),
$$

and by using the natural pairing

$$
H_{\mathrm{DR}}^{2(d-r)+\nu}(X / k) \otimes H_{\mathrm{DR}}^{2 r-\nu}(X / k) \rightarrow H_{\mathrm{DR}}^{2 d}(X / k) \simeq k,
$$

we arrive at $\langle,\rangle_{W, X}^{(\nu)}$. The above properties are now verified by the same argument as that in [Sa1] $\S 7$.

Proof of Theorem 4.2. Let $W, X, \Gamma$ be as in 4.2 and assume $r k_{\mathrm{DR}}^{(\nu)}(\Gamma) \leq \nu-1$. Since the assertion does not specify a ground field, we may select $k \in \mathcal{F}$ so that $X$ is defined over $k$ and the following further conditions hold:

(a) There exists $X_{o}, W_{o} \in \mathcal{C}_{k}$ and $\Gamma \in \mathrm{CH}^{r}\left(W_{o} \times X_{o}, \mathbb{Q}\right)$ which give rise to $W, X, \Gamma$ in 4.2 respectively by the base change via $k \rightarrow \mathbb{C}$.

(b) $W_{o}$ has a $k$-rational point $a$.

(c) There exists $\left\{f_{i}: Y_{o i} \rightarrow X_{o}\right\}_{i \in \mathbb{N}}$, a countable set of morphisms in $\mathcal{C}_{k}$ such that $\operatorname{dim} Y_{o i} \leq m+\nu-1$ for all $i$ and

$$
\Gamma_{*}\left(A_{0}(W)\right) \subset \Xi \stackrel{\text { def }}{=} \operatorname{Image}\left\{\bigoplus_{i \in \mathbb{N}} \mathrm{CH}_{m}\left(Y_{i} ; \mathbb{Q}\right) \stackrel{f_{*}}{\rightarrow} \mathrm{CH}^{r}(X, \mathbb{Q})\right\}+F_{\mathrm{DR} / k}^{\nu+1} \mathrm{CH}^{r}(X / \mathbb{C}) . \quad\left(Y_{i}=Y_{o i} \times{ }_{k} \mathbb{C}\right)
$$

Let $\eta \in W_{o}$ be the generic point and put $K=k(\eta)$ and

$$
\xi:=\Gamma \bullet\left(\eta \times X_{o}\right)-\Gamma \bullet\left(a \times X_{o}\right) \in \mathrm{CH}^{r}\left(X_{o K} ; \mathbb{Q}\right) .
$$

Theorem 4.2 follows from Lemma 4.3 if we show:

$$
\xi \in \operatorname{Image}\left\{\bigoplus_{i \in \mathbb{N}} \mathrm{CH}_{m}\left(Y_{o i K} ; \mathbb{Q}\right) \stackrel{f_{K *}}{\longrightarrow} \mathrm{CH}^{r}\left(X_{o K}, \mathbb{Q}\right)\right\}+F_{\mathrm{DR} / k}^{\nu+1} \mathrm{CH}^{r}\left(X_{o K} / K\right) . \quad\left(Y_{o i K}=Y_{o i} \times_{k} K\right)
$$

Take an embedding $\iota: K \hookrightarrow \mathbb{C}$ of $k$-algebras, which is possible by the assumption $k \in \mathcal{F}$, and let $\xi_{\mathbb{C}} \in$ $\mathrm{CH}^{r}(X ; \mathbb{Q})$ be obtained from $\xi$ by the base change. By definition $\xi_{\mathbb{C}} \in \Gamma_{*}\left(A_{0}(W)\right)$ and hence $\xi_{\mathbb{C}} \in \Xi$ by $(c)$. To deduce $(*)$ from this, we consider the commutative diagram

$$
\begin{array}{ccc}
\bigoplus_{i \in \mathbb{N}} \mathrm{CH}_{m}\left(Y_{o i K} ; \mathbb{Q}\right) & \stackrel{f_{K *}}{\longrightarrow} & \frac{\mathrm{CH}_{m}\left(X_{o K} ; \mathbb{Q}\right)}{F_{\mathrm{DR} / k}^{\nu+1} \mathrm{CH}_{m}\left(X_{o K} / K\right)} \\
\downarrow \iota^{*} & & \downarrow \iota^{*} \\
\bigoplus_{i \in \mathbb{N}} \mathrm{CH}_{m}\left(Y_{i} ; \mathbb{Q}\right) & \stackrel{f_{\mathbb{C} *}}{\longrightarrow} & \frac{\mathrm{CH}_{m}(X ; \mathbb{Q})}{F_{\mathrm{DR} / k}^{\nu+1} \mathrm{CH}_{m}(X / \mathbb{C})}
\end{array}
$$

It suffices to show the injectivity of the induced map $\operatorname{coker}\left(f_{K *}\right) \rightarrow \operatorname{coker}\left(f_{\mathbb{C}_{*}}\right)$. This is shown by the same argument as [Sa1], Lemma (2.9), which we recall for convenience of the readers. Let $\mathcal{S}_{k}$ be as in $\S 3$. For $S \in \mathcal{S}_{k}$ we put

$$
\Lambda_{S}=\operatorname{coker}\left\{\bigoplus_{i \in \mathbb{N}} \mathrm{CH}_{m}\left(Y_{o i S} ; \mathbb{Q}\right) \stackrel{f_{S *}}{\longrightarrow} \frac{\mathrm{CH}_{m}\left(X_{o S} ; \mathbb{Q}\right)}{F_{\mathrm{DR} / k}^{\nu+1} \mathrm{CH}_{m}\left(X_{o S} / S\right)}\right\} .
$$

It suffices to show the injectivity of the induced map $g^{*}: \Lambda_{K} \rightarrow \Lambda_{S}$ for any morphism $g: S \rightarrow \operatorname{Spec}(K)$ in $\mathcal{S}_{k}$, where for notational convenience $\Lambda_{K}$ means $\Lambda_{\operatorname{Spec}(K)}$. We may assume $g$ is of finite type, for if $S=\operatorname{Spec}(L)$ for a finite field extension $L$ of $K$, then we have the functorial map $g_{*}: \Lambda_{L} \rightarrow \Lambda_{K}$ such that $g_{*} g^{*}$ is multiplication by the degree of $L / K$, which implies the desired injectivity. Here we used Lemma 3.6. In general, by what is just shown, we may assume that there exists a section $i: \operatorname{Spec}(K) \rightarrow S$ of $g$. Then the composite $\Lambda_{K} \stackrel{g^{*}}{\longrightarrow} \Lambda_{S} \stackrel{i^{*}}{\rightarrow} \Lambda_{K}$ is the identity and hence $g^{*}$ is injective. This completes the proof of Theorem 4.2 .

Recall $\left\{F_{B}^{\nu} \mathrm{CH}^{r}(X ; \mathbb{Q})\right\}_{\nu \geq 0}$ as introduced earlier in Definition 2.4 of $\S 2$. Theorem 0.2 follows from the following: 
Corollary 4.4. Let $X \in \mathcal{C}$ with $\operatorname{dim} X=d$. Assume that either $\nu \leq 2$ or that there exists a dominant rational morphism $\tilde{X} \rightarrow X$ such that the condition $B(\tilde{X})$ holds $(c f . \S 1(v))$. Then

$$
r k_{\mathrm{DR}}^{(\nu)}\left(F_{B}^{\nu} C H_{0}(X ; \mathbb{Q})\right) \leq \nu-1 \Rightarrow H^{0}\left(X, \Omega_{X / \mathbb{C}}^{\nu}\right)=0 .
$$

Proof. This is an immediate consequence of Theorem 4.2 applied to the case $W=X$ and $\Gamma=\Delta_{X}$, together with the following result ([Sa1], Theorem (6.2)).

Theorem 4.5. Let the assumption be as in 4.4. Then there exists a morphism $f: Y \rightarrow X$ in $\mathcal{C}$ such that $\operatorname{dim} Y \leq \nu-1$ and

$$
A_{0}(X) \subset F_{B}^{\nu} C H_{0}(X ; \mathbb{Q})+f_{*}\left(A_{0}(Y)\right) .
$$

Theorem 0.3 follows from the following result:

Corollary 4.6. Let $X \subset \mathbb{P}^{d+1}$ be a general hypersurface of degree $m \geq 3$ over $\mathbb{C}$, where "general" means corresponding to a point in a certain nonempty Zariski open subset of the universal family of hypersurfaces of degree $m$ in $\mathbb{P}^{d+1}$. Let $k=\left[\frac{d+1}{m}\right]$ (greatest integer) and $r=d-k$ and $\nu=d-2 k$, and assume that the following numerical condition holds:

$$
k(d+2-k)+1-\left(\begin{array}{c}
m+k \\
k
\end{array}\right) \geq 0
$$

Then $r k_{\mathrm{DR}}^{(\nu)}\left(A^{r}(X)\right)=\nu$ (Note that $A^{r}(X) \subset F_{B}^{\nu} C H^{r}(X)$ by Proposition 2.7).

Proof. Let $\mathcal{G}_{X}(k)$ be the moduli space of the linear subspaces of dimension $k$ contained in $X$. Then there exists a smooth projective subvariety $W \subset \mathcal{G}_{X}(k)$, of dimension $\nu$, such that the induced cylinder map

$$
[\Gamma]_{*}: H^{d-2 k}(W, \mathbb{C}) \rightarrow H^{d}(X, \mathbb{C}),
$$

is surjective, where $\Gamma \in \mathrm{CH}^{r}(W \times X)$ is the incidence correspondence ([Le3]). The numerical condition implies $H^{k}\left(X, \Omega_{X / \mathbb{C}}^{d-k}\right) \neq 0$ (see $[$ Le1] $)$ so that Image $\left([\Gamma]_{*}\right) \not \subset F^{k+1} H^{d}(X, \mathbb{C})$. It implies Image $\left([\Gamma]^{*}\right) \not \subset F^{1} H^{\nu}(W, \mathbb{C})$, where $[\Gamma]^{*}: H^{d}(X, \mathbb{C}) \rightarrow H^{\nu}(W, \mathbb{C})$ is the dual of $[\Gamma]_{*}$ ([Sa1], Cor.(8.4)). It follows from Theorem 4.2 that $r k_{\mathrm{DR}}^{(\nu)}(\Gamma)=\nu$. In fact, it is also the case that $\Gamma_{*}\left(A_{0}(W)\right)=A^{r}(X)([\operatorname{Le} 3])$, thus $r k_{\mathrm{DR}}^{(\nu)}\left(A^{r}(X)\right)=\nu$.

Remark 4.7. The assumption of $X$ being general in Corollary 4.6 is needed in [Le3] to arrive at results on the level of Chow groups, in particular our above assertion $\Gamma_{*}\left(A_{0}(W)\right)=A^{r}(X)$. For instance, we need (among other things), the nonsingularity and predicted dimension of the Fano variety of $k$-planes on $X$. 


\section{§5. Cycle Classes in higher extension groups}

In this section we give a brief explanation of cycle classes in higher extension groups in suitable categories following the works of Green-Griffiths, M. Asakura and M. Saito (cf. [Gr], [As1] and [MSa2]). It is motivated by Beilinson's conjectural formula in $\S 0$. Fix a base field $k \subset \mathbb{C}$ as in the previous sections.

5.1. Mumford-Griffiths invariants as higher extensions. Let $R$ be a localization of a smooth algebra over $k$ and let $\mathrm{D}_{R} \subset \operatorname{End}_{k}(R)$ be the ring of differential operators generated as a subring by $\Theta_{R}=\operatorname{Der}_{k}(R, R)$ (derivations) and $R$ (scalars). If $R$ has a local coordinate $\left\{x_{i}\right\}_{1 \leq i \leq n}$ and $\left\{\partial_{i}\right\}_{1 \leq i \leq n}$ is the dual basis of $\left\{d x_{i}\right\}_{1 \leq i \leq n}$, we have

$$
\mathrm{D}_{R}=\bigoplus_{\alpha \in \mathbb{Z}_{+}^{n}} R \cdot \partial^{\alpha} \quad\left(\partial^{\alpha}:=\partial_{1}^{\alpha_{1}} \partial_{2}^{\alpha_{2}} \cdots \partial_{n}^{\alpha_{n}}\right) .
$$

It is endowed with the filtration of differential order:

$$
F_{p} \mathrm{D}_{R}=\bigoplus_{|\alpha| \leq p} R \cdot \partial^{\alpha} \quad\left(|\alpha|=\sum_{i=1}^{n} \alpha_{i}\right) .
$$

A filtered $\mathrm{D}_{R}$-module is a pair $(M, F)$ consisting of a $\mathrm{D}_{R}$-module $M$ and an increasing filtration of finite $R$-modules $F_{p} M \subset M(p \in \mathbb{Z})$ satisfying

(1) $M=\bigcup_{p \in \mathbb{Z}} F_{p} M \quad\left(F_{p} M=0 \quad p<<0\right)$,

(2) $F_{p} \mathrm{D}_{R} \cdot F_{q} M \subset F_{p+q} M$.

Let $\mathcal{M F}(R)$ be the category of filtered $\mathrm{D}_{R}$-modules. For an object $M=(M, F)$ of $\mathcal{M F}(R)$ its Tate twist $M(r)$ is defined to be $(M, F(r))$ with $F(r)_{p}=F_{p-r}$. Let $R(m) \in \mathcal{M F}(R)$ be the $\mathrm{D}_{R}$-module $R$ endowed with the filtration $F_{p} R=0$ if $p<m$ and $F_{p} R=R$ if $p \geq m$. $\mathcal{M F}(R)$ becomes an exact category by defining a complex in $\mathcal{M F}(R)$

$$
\left(M_{1}, F_{1}\right) \rightarrow\left(M_{2}, F_{2}\right) \rightarrow\left(M_{2}, F_{2}\right)
$$

to be exact if and only if

$$
G r^{F_{1}} M_{1} \rightarrow G r^{F_{2}} M_{2} \rightarrow G r^{F_{3}} M_{3}
$$

is an exact sequence of $R$-modules. Thus we can consider higher extension groups in the sense of Yoneda in $\mathcal{M F}(R)$.

Let $f: X \rightarrow S=\operatorname{Spec}(R)$ be a smooth projective morphism. The de Rham cohomology $M=$ $H_{\mathrm{DR}}^{q}(X / S)=\mathbb{H}^{q}\left(\Omega_{X / S}^{\bullet}\right)$ with the filtration $F_{p} M:=F^{-p} H_{\mathrm{DR}}^{q}(X / S)$ gives rise to an object of $\mathcal{M F}(R)$ : We let $\theta \in \Theta_{R}$ act on $M$ via $\nabla_{\theta}$, the covariant derivative of $\theta$ with respect to the arithmetic Gauss-Manin connection

$$
\nabla: H_{\mathrm{DR}}^{q}(X / S) \rightarrow \Omega_{R / k}^{1} \otimes H_{\mathrm{DR}}^{q}(X / S) .
$$

Proposition 5.2. (cf. [Gr] and [As1], 3.2) For integers $p, q \geq 0, \operatorname{Ext}_{\mathcal{M F}(R)}^{p}\left(R(0), H_{D R}^{q}(X / S)(r)\right)$ is isomorphic to the cohomology of the following complex

$$
\Omega_{R / k}^{p-1} \otimes F^{r-p+1} H_{\mathrm{DR}}^{q}(X / S) \stackrel{\nabla}{\rightarrow} \Omega_{R / k}^{p} \otimes F^{r-p} H_{\mathrm{DR}}^{q}(X / S) \stackrel{\nabla}{\rightarrow} \Omega_{R / k}^{p+1} \otimes F^{r-p-1} H_{\mathrm{DR}}^{q}(X / S) .
$$


Corollary 5.3. We recall that $S=\operatorname{Spec}(R)$. For integers $r, \nu \geq 0$ there is a canonical isomorphism

$$
\nabla J^{r, \nu}(X / S) \simeq \operatorname{Ext}_{\mathcal{M F}(R)}^{\nu}\left(R(0), H_{\mathrm{DR}}^{2 r-\nu}(X / S)(r)\right) .
$$

5.4. Arithmetic mixed Hodge modules. In the above construction, the $\mathbb{Q}$-structure is not taken into account. The theory of arithmetic mixed Hodge modules remedies the defect and gives a refinement of the MumfordGriffiths invariants.

For a smooth variety $X$ over $k$, the category $\operatorname{MHM}(X)$ of mixed Hodge modules on $X$ was defined by Morihiko Saito $([\mathrm{MSa} 3])$. Let $X(\mathbb{C})$ be the set of $\mathbb{C}$-valued points over $k$ endowed with the usual analytic topology. An object of $\operatorname{MHM}(X)$, called a mixed Hodge module on $X$, is given by a triple $\left(K_{\mathbb{Q}},(M, F), \iota\right)$ satisfying certain properties, where $K_{\mathbb{Q}}$ is a perverse sheaf of $\mathbb{Q}$-vector spaces of finite rank on $X(\mathbb{C})$, and $(M, F)$ is a filtered $D_{X}$-module which is holonomic and has regular singularities, and $\iota$ is a quasi-isomorphism (Riemann-Hilbert correspondence)

$$
D R(M) \simeq K_{\mathbb{Q}} \otimes \mathbb{C} \quad \text { with } D R(M)=M \otimes_{k} \Omega_{X(\mathbb{C})}^{\bullet}[\operatorname{dim}(X)] .
$$

(To be more precise, a mixed Hodge module is equipped with a weight filtration. Since it is not used in this paper, we have omitted it for the sake of simplicity. The reader may consult the brief exposition in [AS1] §2.) The category $\operatorname{MHM}(X)$ of mixed Hodge modules on $X$ is an abelian category. For a morphism $f: X \rightarrow Y$ there are the standard operations $f_{*}, f^{*}, f_{!}, f^{!}$, etc., on the derived category of bounded complexes of mixed Hodge modules on $X$ and $Y$. There exists a cycle class map

$$
\rho_{X}^{r}: \operatorname{CH}^{r}(X ; \mathbb{Q}) \rightarrow \operatorname{Ext}_{\operatorname{MHM}(X)}^{2 r}\left(\mathbb{Q}_{X}(0), \mathbb{Q}_{X}(r)\right),
$$

where $\mathbb{Q}_{X}(r)$ is the Tate object in $\operatorname{MHM}(X)$ whose underlying perverse sheaf is $\mathbb{Q}_{X}[\operatorname{dim}(X)]$ (the constant local system with a degree shift) and whose underlying $D_{X}$-module is $\mathcal{O}_{X}$ with the filtration given by $F_{-r} \mathcal{O}_{X}=\mathcal{O}_{X}$ and $F_{-r-1} \mathcal{O}_{X}=0$.

Let $f: X \rightarrow S$ be a morphism of quasiprojective smooth varieties over $k$. The standard operations provide us with the Leray spectral sequence

$$
E_{2}^{p, q}=\operatorname{Ext}_{\operatorname{MHM}(S)}^{p}\left(\mathbb{Q}_{S}(0), R^{q} f_{*} \mathbb{Q}_{X}(r)\right) \Rightarrow \operatorname{Ext}_{\operatorname{MHM}(X)}^{p+q}\left(\mathbb{Q}_{X}(0), \mathbb{Q}_{X}(r)\right),
$$

where $R^{q} f_{*}: \operatorname{MHM}(X) \rightarrow \operatorname{MHM}(S)$ is the derived functor on the categories of mixed Hodge modules. The decomposition theorem for mixed Hodge modules implies that the spectral sequence degenerates at the $E_{2}$-term, provided $f$ is proper.

In what follows we assume further that $f$ is proper and smooth. Letting

$$
F_{L}^{\nu} \operatorname{Ext}_{\operatorname{MHM}(X)}^{2 r}\left(\mathbb{Q}_{X}(0), \mathbb{Q}_{X}(r)\right) \subset \operatorname{Ext}_{\operatorname{MHM}(X)}^{2 r}\left(\mathbb{Q}_{X}(0), \mathbb{Q}_{X}(r)\right)
$$

be the filtration inducing the spectral sequence, we can show

$$
\rho_{X}^{r}\left(F_{B}^{\nu} \mathrm{CH}^{r}(X / S ; \mathbb{Q})\right) \subset F_{L}^{\nu} \operatorname{Ext}_{\operatorname{MHM}(X)}^{2 r}\left(\mathbb{Q}_{X}(0), \mathbb{Q}_{X}(r)\right)
$$

by the same argument as in [Sa3], Prop.(2-1) (also see the proof of Prop.3.7). Hence we get the induced map

$$
\rho_{X / S}^{r, \nu}: G_{F}^{\nu} \mathrm{CH}^{r}(X / S ; \mathbb{Q}) \rightarrow \operatorname{Ext}_{\mathrm{MHM}(S)}^{\nu}\left(\mathbb{Q}_{S}(0), R^{2 r-\nu} f_{*} \mathbb{Q}_{X}(r)\right) .
$$

If $S=\operatorname{Spec}(R)$, there is a natural functor

$$
r_{\mathcal{M F}}: \operatorname{MHM}(S) \rightarrow \mathcal{M F}(R)
$$


which sends a mixed Hodge module to its underlying filtered $D_{S}$-module. We have $r_{\mathcal{M F}}\left(\mathbb{Q}_{S}(m)\right)=R(m)$ and $r_{\mathcal{M F}}\left(R^{q} f_{*} \mathbb{Q}_{X}(r)\right)=H_{\mathrm{DR}}^{q}(X / S)(r)$ and the map $\phi_{X / S}^{r, \nu}$ in Proposition 3.7 coincides with the composite of $\rho_{X / S}^{r, \nu}$ and the map induced by $r_{\mathcal{M F}}$ :

$$
\operatorname{Ext}_{\operatorname{MHM}(S)}^{\nu}\left(\mathbb{Q}_{S}(0), R^{2 r-\nu} f_{*} \mathbb{Q}_{X}(r)\right) \rightarrow \operatorname{Ext}_{\mathcal{M F}(R)}^{\nu}\left(R(0), H_{\mathrm{DR}}^{2 r-\nu}(X / S)(r)\right) \simeq \nabla J^{r, \nu}(X / S),
$$

where the last isomorphism is given in Corollary 5.3.

Let $\eta: \operatorname{Spec}(\mathbb{C}) \rightarrow S$ be a $k$-morphism and $X_{\eta}=X \times_{S} \operatorname{Spec}(\mathbb{C})$ be the base change. Noting that $\operatorname{MHM}(\operatorname{Spec}(\mathbb{C}))$ is the category MHS of graded polarized $\mathbb{Q}$-mixed Hodge strucutres, $\eta$ induces a natural functor

$$
r_{H, \eta}: \operatorname{MHM}(S) \rightarrow \mathrm{MHS} .
$$

It satisfies

$$
r_{H, \eta}\left(\mathbb{Q}_{S}(m)\right)=\mathbb{Q}(m) \text { and } r_{H, \eta}\left(R^{q} f_{*} \mathbb{Q}_{X}(r)\right)=H^{q}\left(X_{\eta, a n}, \mathbb{Q}(r)\right),
$$

and the composite of $\rho_{X}^{r, 1}$ with the map

$$
\operatorname{Ext}_{\mathrm{MHM}(S)}^{1}\left(\mathbb{Q}_{S}(0), R^{2 r-1} f_{*} \mathbb{Q}_{X}(r)\right) \rightarrow \operatorname{Ext}_{\mathrm{MHS}}^{1}\left(\mathbb{Q}(0), H^{2 r-1}\left(X_{\eta, a n}, \mathbb{Q}(r)\right)\right) \simeq J^{r}\left(X_{\eta}\right),
$$

coincides with the Griffiths Abel-Jacobi map for $X_{\eta}$, where the first map is induced by $r_{H, \eta}$ and the second map is Carlson's isomorphism ([Ca]).

Remark 5.5. As pointed out by one of the referees, the map induced by $r_{\mathcal{M F}}$ into $\nabla J^{r, 1}(X / S)$ functions as an infinitesimal invariant for the map induced by $r_{H, \eta}$ (as $\eta$ varies). For $\nu>1, r_{\mathcal{M F}}$ 's induced map serves as a "higher infinitesimal invariant". 


\section{§6. A map from Cycle Classes to De Rham/Mumford-Griffiths invariants}

In order to prove Theorem 0.4, we need cycle classes which capture cycles with trivial Mumford-Griffiths invariants. In this section we construct it by using the arithmetic cycle classes in higher extension groups in the category of arithmetic mixed Hodge modules. We then apply these ideas to to a particular setting in $\S 7$, by proving Theorem 7.2 below, which in turn implies Theorem 0.4.

Let the notation be as in $\S 5$. Let $f: X \rightarrow S$ be a proper smooth morphism of quasiprojective smooth varieties over $k$ and let $g: S \rightarrow \operatorname{Spec}(k)$ be the natural morphism. Recall the notation in $\S 1$ (iv). For $L \in \operatorname{MHM}(S)$ we have the Leray spectral sequence

$$
E_{2}^{p, q}=\operatorname{Ext}_{\mathrm{MHM}(\operatorname{Spec}(k))}^{p}\left(\mathbb{Q}(0), R^{q} g_{*} L\right) \Rightarrow \operatorname{Ext}_{\mathrm{MHM}(S)}^{p+q}\left(\mathbb{Q}_{S}(0), L\right)
$$

associated to the derived functor $R g_{*}: D^{b}(\operatorname{MHM}(S)) \rightarrow D^{b}(\operatorname{MHM}(\operatorname{Spec}(k)))$. Note that $\operatorname{MHM}(\operatorname{Spec}(k))$ is identified with a subcategory of MHS via the functor $r_{H, \eta}$ (5.4.1) with $\eta: \operatorname{Spec}(\mathbb{C}) \rightarrow \operatorname{Spec}(k)$ induced by the natural embedding $k \hookrightarrow \mathbb{C}$, and $\mathbb{Q}(0)$ denotes the usual Hodge structure. Noting that $\operatorname{Ext}_{\mathrm{MHM}(\operatorname{Spec}(k))}^{p}=0$ for $p \geq 2$, we get the short exact sequence

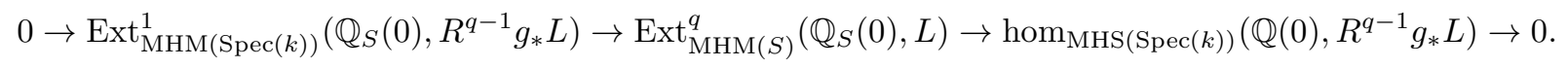

Apply this to $L=R^{2 r-\nu} f_{*} \mathbb{Q}_{X}(r) \in \operatorname{MHM}(S)$ and we get natural maps

$$
\begin{gathered}
\pi: \operatorname{Ext}_{\mathrm{MHM}(S)}^{\nu}\left(\mathbb{Q}_{S}(0), R^{2 r-\nu} f_{*} \mathbb{Q}_{X}(r)\right) \rightarrow \Lambda^{r, \nu}(X / S):=\operatorname{hom}_{\mathrm{MHS}}\left(\mathbb{Q}(0), H^{\nu}\left(S / \mathbb{C}, R^{2 r-\nu} f_{*} \mathbb{Q}(r)\right)\right), \\
\operatorname{ker}(\pi) \rightarrow \Xi^{r, \nu}(X / S):=\operatorname{Ext}_{\mathrm{MHS}}^{1}\left(\mathbb{Q}(0), H^{\nu-1}\left(S / \mathbb{C}, R^{2 r-\nu} f_{*} \mathbb{Q}(r)\right)\right),
\end{gathered}
$$

where $\left.H^{\bullet}\left(S / \mathbb{C}, R^{2 r-\nu} f_{*} \mathbb{Q}(r)\right)\right)$, the cohomology of $S_{a n}$ with coefficients in a local system, is endowed in a canonical way with a mixed Hodge structure by the theory of mixed Hodge modules ([MSa1]). The composite of $\rho_{X / S}^{r, \nu}$ and $\pi$

$$
\lambda_{X / S}^{r, \nu}: F_{B}^{\nu} \mathrm{CH}^{r}(X / S ; \mathbb{Q}) \rightarrow \Lambda^{r, \nu}(X / S)
$$

is given as follows. Let

$$
c_{X}^{r}: \mathrm{CH}^{r}(X ; \mathbb{Q}) \rightarrow H^{2 r}(X / \mathbb{C}, \mathbb{Q}(r))
$$

be the cycle class map. We have $c_{X}^{r}\left(F_{B}^{\nu} \mathrm{CH}^{r}(X / S ; \mathbb{Q})\right) \subset F_{L}^{\nu} H^{2 r}(X / \mathbb{C}, \mathbb{Q}(r)$ ), (again, by the same argument as [Sa3], Prop. 2.1), where $F_{L}^{\nu}$ denotes the filtration inducing the Leray spectral sequence

$$
E_{2}^{p, q}=H^{p}\left(S / \mathbb{C}, R^{q} f_{*} \mathbb{Q}(r)\right) \Rightarrow H^{p+q}(X / \mathbb{C}, \mathbb{Q}(r)),
$$

which degenerates at $E_{2}$ by Deligne's criterion ([De3]). Thus the projection to the graded quotient induces

$$
F_{B}^{\nu} \mathrm{CH}^{r}(X / S ; \mathbb{Q}) \rightarrow H^{\nu}\left(S / \mathbb{C}, R^{2 r-\nu} \rho_{*} \mathbb{Q}(r)\right)
$$

which is identified with $\lambda_{X / S}^{r, \nu}$ composed with $\Lambda^{r, \nu}(X / S) \hookrightarrow H^{\nu}\left(S / \mathbb{C}, R^{2 r-\nu} \rho_{*} \mathbb{Q}(r)\right)$, the natural inclusion. In particular we get the natural map

$$
\epsilon_{X / S}^{r, \nu}: F_{B}^{\nu} \mathrm{CH}^{r}(X / S ; \mathbb{Q}) \cap \mathrm{CH}_{\mathrm{hom}}^{r}(X ; \mathbb{Q}) \rightarrow \Xi^{r, \nu}(X / S)
$$

for which we have

$$
\epsilon_{X / S}^{r, \nu}\left(F_{B}^{\nu+1} \mathrm{CH}^{r}(X / S ; \mathbb{Q}) \cap \mathrm{CH}_{\mathrm{hom}}^{r}(X ; \mathbb{Q})\right)=0 .
$$

The reader can also consult [Le2] for the similar construction in terms of absolute Hodge cohomology. 
Proposition 6.3. (i) Assume $S=\operatorname{Spec}(R)$ is an affine variety. There is an injective map

$$
\Phi_{X / S}^{r, \nu}: \Lambda^{r, \nu}(X / S) \hookrightarrow \nabla D R^{r, \nu}(X / S) \otimes_{k} \mathbb{C},
$$

which fits into the commutative diagram

$$
\begin{array}{ccc}
F_{B}^{\nu} C H^{r}(X / S ; \mathbb{Q}) & \stackrel{\phi_{X / S, \mathrm{DR}}^{r, \nu}}{\longrightarrow} & \nabla D R^{r, \nu}(X / S) \\
\downarrow \lambda_{X / S}^{r, \nu} & & \downarrow \\
\Lambda^{r, \nu}(X / S) & \stackrel{\Phi_{X / S}^{r, \nu}}{\longrightarrow} & \nabla D R^{r, \nu}(X / S) \otimes_{k} \mathbb{C}
\end{array}
$$

where $\phi_{X / S, \mathrm{DR}}^{r, \nu}$ is defined in (3.7.1).

(ii) Suppose that $S$ and $X$ are projective, and $U \subset S$ is an open affine subvariety over $k$. Then there is a map

$$
\Phi_{X / U}^{r, \nu}: \Lambda^{r, \nu}(X / S) \rightarrow \nabla J^{r, \nu}\left(X_{U} / U\right) \otimes_{k} \mathbb{C}, \quad\left(X_{U}=X \times_{S} U\right)
$$

which fits into the commutative diagram

$$
\begin{array}{ccc}
F_{B}^{\nu} C H^{r}(X / S ; \mathbb{Q}) & \stackrel{\phi_{X}^{r, \nu} / U}{\longrightarrow} & \nabla J^{r, \nu}\left(X_{U} / U\right) \\
\downarrow \lambda_{X / S}^{r, \nu} & & \downarrow \\
\Lambda^{r, \nu}(X / S) & \stackrel{\Phi_{X / U}^{r, \nu}}{\longrightarrow} & \nabla J^{r, \nu}\left(X_{U} / U\right) \otimes_{k} \mathbb{C}
\end{array}
$$

Proof. Part (i). Recall the notation in $\S 1$ (iv). The complex of holomorphic forms on $S / \mathbb{C}$ with values in $R^{2 r-\nu} f_{*} \mathbb{C}$ furnishes a resolution of $R^{2 r-\nu} f_{*} \mathbb{C}$. Since $S$ is affine, a variant of the Grothendieck Algebraic de Rham Theorem implies that $H^{\nu}\left(S / \mathbb{C}, R^{2 r-\nu} f_{*} \mathbb{C}\right)$ can be computed from algebraic differential forms ([De2] (§6, pp. 98-99)). Specifically

$$
H^{\nu}\left(S / \mathbb{C}, R^{2 r-\nu} f_{*} \mathbb{C}\right) \simeq \mathbb{H}^{\nu}\left(\Omega_{S / k}^{\bullet} \otimes_{\mathcal{O}_{S}} \mathbb{R}^{2 r-\nu} f_{*} \Omega_{X / S}^{\bullet}\right) \otimes_{k} \mathbb{C}
$$

where the latter term is computed in the Zariski topology. Since $S$ is affine and $\Omega_{S / k}^{\bullet} \otimes_{\mathcal{O}_{S}} \mathbb{R}^{2 r-\nu} f_{*} \Omega_{X / S}^{\bullet}$ is coherent, $\mathbb{H}^{\nu}\left(\Omega_{S / k}^{\bullet} \otimes_{\mathcal{O}_{S}} \mathbb{R}^{2 r-\nu} f_{*} \Omega_{X / S}^{\bullet}\right)$ is computed as

$$
\frac{\operatorname{ker}\left(\nabla: H^{0}\left(S, \Omega_{S / k}^{\nu} \otimes \mathbb{R}^{2 r-\nu} f_{*} \Omega_{X / S}^{\bullet}\right) \rightarrow H^{0}\left(S, \Omega_{S / k}^{\nu+1} \otimes \mathbb{R}^{2 r-\nu} f_{*} \Omega_{X / S}^{\bullet}\right)\right)}{\nabla\left(H^{0}\left(S, \Omega_{S / k}^{\nu-1} \otimes \mathbb{R}^{2 r-\nu} f_{*} \Omega_{X / S}^{\bullet}\right)\right)},
$$

which coincides with $\nabla D R^{r, \nu}(X / S)$ by definition (Def. 3.2). Thus we have

$$
\Lambda^{r, \nu}(X / S) \subset H^{\nu}\left(S / \mathbb{C}, R^{2 r-\nu} f_{*} \mathbb{C}\right) \simeq \nabla D R^{r, \nu}(X / S) \otimes_{k} \mathbb{C},
$$

which defines the injection $\Lambda^{r, \nu}(X / S) \hookrightarrow \nabla D R^{r, \nu}(X / S) \otimes_{k} \mathbb{C}$.

Part (ii). From the work of Deligne (see [B-Z]), the complex

$$
\left(\Omega_{S / k}^{\bullet} \otimes \mathbb{R}^{2 r-\nu} f_{*} \Omega_{X_{\mathbb{C}} / S_{\mathbb{C}}}^{* \geq r-\bullet}, \nabla\right)
$$


computes $F^{r} H^{\nu}\left(S / \mathbb{C}, R^{2 r-\nu} f_{*} \mathbb{C}\right)$. This is in the strong topology, but using the second spectral sequence on hypercohomology given in $[\mathrm{G}-\mathrm{H}]$ (p. 446):

$$
{ }^{\prime \prime} E_{2}^{\bullet \bullet \bullet}=H_{\nabla}^{\bullet}\left(H^{\bullet}\left(\Omega_{S / k}^{\bullet} \otimes \mathbb{R}^{2 r-\nu} f_{*} \Omega_{X_{\mathbb{C}} / S_{\mathbb{C}}}^{* \geq r-\bullet}\right)\right) \Rightarrow \mathbb{H}^{\bullet}\left(\Omega_{S / k}^{\bullet} \otimes \mathbb{R}^{2 r-\nu} f_{*} \Omega_{X_{\mathbb{C}} / S_{\mathbb{C}}}^{* \geq r-\bullet}\right),
$$

and the assumed projectivity of $S$, together with Serre's work (GAGA), the same result holds in the Zariski topology. Specifically, we get

$$
F^{r} H^{\nu}\left(S / \mathbb{C}, R^{2 r-\nu} f_{*} \mathbb{C}\right) \simeq \mathbb{H}^{\nu}\left(\Omega_{S / k}^{\bullet} \otimes \mathbb{R}^{2 r-\nu} f_{*} \Omega_{X / S}^{* \geq r-\bullet}\right) \otimes_{k} \mathbb{C} .
$$

Next, by restriction there is a map

$$
\begin{gathered}
\mathbb{H}^{\nu}\left(\Omega_{S / k}^{\bullet} \otimes \mathbb{R}^{2 r-\nu} f_{*} \Omega_{X / S}^{* \geq r-\bullet}\right) \rightarrow \mathbb{H}^{\nu}\left(\Omega_{U / k}^{\bullet} \otimes \mathbb{R}^{2 r-\nu} f_{*} \Omega_{X_{U} / U}^{* \geq r-\bullet}\right) \\
\simeq \frac{\operatorname{ker}\left(\nabla: H^{0}\left(U, \Omega_{U / k}^{\nu} \otimes \mathbb{R}^{2 r-\nu} f_{*} \Omega_{X_{U} / U}^{\bullet>r-\nu}\right) \rightarrow H^{0}\left(S, \Omega_{U / k}^{\nu+1} \otimes \mathbb{R}^{2 r-\nu} f_{*} \Omega_{X_{U} / U}^{\bullet r-1}\right)\right)}{\nabla\left(H^{0}\left(U, \Omega_{U / k}^{\nu-1} \otimes \mathbb{R}^{2 r-\nu} f_{*} \Omega_{X_{U} / U}^{\bullet r-\nu+1}\right)\right)},
\end{gathered}
$$

where the isomorphism uses the fact that $U$ is affine. The last term is identified with $\nabla J^{r, \nu}\left(X_{U} / U\right)$ by Def. 3.2. This gives rise to the desired map.

It would be nice if one could have Proposition 6.3 (ii) without assuming $X$ and $S$ are projective. Indeed we will provide strong evidence in support of the following:

Conjecture 6.4. Put

$$
\Lambda_{\mathrm{alg}}^{r, \nu}(X / S)=\operatorname{Image}\left(F_{B}^{\nu} C H^{r}(X / S ; \mathbb{Q}) \stackrel{\lambda_{X / S}^{r, \nu}}{\longrightarrow} \Lambda^{r, \nu}(X / S) .\right.
$$

Then, for any dense affine open $U \subset S$, there is a map

$$
\Phi_{X / U}^{r, \nu}: \Lambda_{\text {alg }}^{r, \nu}(X / S) \rightarrow \nabla J^{r, \nu}\left(X_{U} / U\right) \otimes_{k} \mathbb{C}, \quad\left(X_{U}=X \times_{S} U\right)
$$

which fits into the commutative diagram

$$
\begin{array}{ccc}
F_{B}^{\nu} C H^{r}(X / S ; \mathbb{Q}) & \stackrel{\phi_{X U}^{r, \nu} / U}{\longrightarrow} & \nabla J^{r, \nu}\left(X_{U} / U\right) \\
\downarrow \lambda_{X / S}^{r, \nu} & & \downarrow \\
\Lambda_{\mathrm{alg}}^{r, \nu}(X / S) & \stackrel{\Phi_{X / U}^{r, \nu}}{\longrightarrow} & \nabla J^{r, \nu}\left(X_{U} / U\right) \otimes_{k} \mathbb{C}
\end{array}
$$

Our approach to the above conjecture involves $L^{2}$-cohomology techniques, where the case $\operatorname{dim} S=1$ has been worked out by Zucker (see [B-Z]). The story for $\operatorname{dim} S>1$ is rather complicated, and is clarified to some degree in the Appendix to this paper. Indeed, the above conjecture is a consequence of other "very reasonable" conjectures.

Choose a morphism $\eta: \operatorname{Spec}(\mathbb{C}) \rightarrow S$ whose image is the generic point and let $X_{\eta}$ be the base change via $\eta$. By definition $X_{\eta}$ is proper smooth over $\mathbb{C}$. Recall $\nabla J^{r, \nu}\left(X_{\eta}\right):=\nabla J^{r, \nu}\left(X_{\eta} / \mathbb{C}\right)$ and $\nabla D R^{r, \nu}\left(X_{\eta}\right):=$ $\nabla D R^{r, \nu}\left(X_{\eta} / \mathbb{C}\right)$ in Definition 3.2. 
Proposition 6.5. (i) There is a map

$$
\Phi_{X_{\eta}}^{r, \nu}: \Lambda^{r, \nu}(X / S) \rightarrow \nabla D R^{r, \nu}\left(X_{\eta}\right) \otimes_{k} \mathbb{C}
$$

which fits into the commutative diagram

$$
\begin{array}{ccc}
F_{B}^{\nu} C H^{r}(X / S ; \mathbb{Q}) & \stackrel{\lambda_{X / S}^{r, \nu}}{\longrightarrow} & \Lambda^{r, \nu}(X / S) \\
\downarrow \eta^{*} & & \downarrow \Phi_{X_{\eta}}^{r, \nu} \\
F_{B}^{\nu} C H^{r}\left(X_{\eta} ; \mathbb{Q}\right) & \stackrel{\phi_{X}^{r, \nu}}{\longrightarrow} \nabla J^{r, \nu}\left(X_{\eta}\right) \otimes_{k} \mathbb{C} \rightarrow & \nabla D R^{r, \nu}\left(X_{\eta}\right) \otimes_{k} \mathbb{C}
\end{array}
$$

In particular we have

$$
\phi_{X_{\eta}, D R}^{r, \nu} \circ \eta^{*}\left(F_{B}^{\nu} C H^{r}(X / S ; \mathbb{Q}) \cap C H_{\mathrm{hom}}^{r}(X ; \mathbb{Q})\right)=0 .
$$

(ii) Suppose that $X$ and $S$ are projective over $k$ or that Conjecture 6.4 is true. Then there is a morphism

$$
\Lambda_{\text {alg }}^{r, \nu}(X / S) \rightarrow \nabla J^{r, \nu}\left(X_{\eta}\right) \otimes_{k} \mathbb{C}
$$

which is compatible with the diagram in 6.5. In particular, we have

$$
\phi_{X_{\eta}}^{r, \nu} \circ \eta^{*}\left(F_{B}^{\nu} C H^{r}(X / S ; \mathbb{Q}) \cap C H_{\mathrm{hom}}^{r}(X ; \mathbb{Q})\right)=0 .
$$

Remarks 6.7. The construction of the map $\Lambda^{r, \nu}(X / S) \rightarrow \nabla J^{r, \nu}\left(X_{\eta}\right) \otimes_{k} \mathbb{C}$, also appears in [Ke1] in the special case where $X=Y \times S$ with $Y, S \in \mathcal{C}_{k}$. In this case, for an affine open subscheme $U \subset S$, we have

$$
\begin{array}{r}
\Lambda^{r, \nu}\left(X \times_{S} U / U\right)=\operatorname{hom}_{\mathrm{MHS}}\left(\mathbb{Q}(0), H^{\nu}(U / \mathbb{C}, \mathbb{Q}) \otimes H^{2 r-\nu}(Y / \mathbb{C}, \mathbb{Q})\right) \hookrightarrow \mathbb{H}^{\nu}\left(\Omega_{U / k}^{\bullet}\right) \otimes F^{r-\nu} H_{\mathrm{DR}}^{2 r-\nu}(Y / k) \otimes_{k} \mathbb{C} \\
=H^{\nu}\left(\Gamma\left(U, \Omega_{U / k}^{\bullet}\right)\right) \otimes F^{r-\nu} H_{\mathrm{DR}}^{2 r-\nu}(Y / k) \otimes_{k} \mathbb{C},
\end{array}
$$

via the comparison isomorphism $H_{\mathrm{DR}}^{\bullet}(Y / k) \otimes_{k} \mathbb{C} \simeq H^{\bullet}(Y / \mathbb{C}, \mathbb{C})$ and Grothendieck's algebraic de Rham theorem. One then applies the morphism

$$
H^{\nu}\left(\Gamma\left(U, \Omega_{U / k}^{\bullet}\right)\right) \otimes F^{r-\nu} H_{\mathrm{DR}}^{2 r-\nu}(Y / k) \otimes_{k} \mathbb{C} \rightarrow H_{\nabla}^{\nu}\left(\Omega_{U / k}^{\bullet} \otimes F^{r-\bullet} H_{\mathrm{DR}}^{2 r-\nu}(Y / k)\right) \otimes_{k} \mathbb{C} .
$$

\section{§7. Detecting nonzero Classes with trivial de Rham/Mumford invariant}

In this section we take the base field $k=\overline{\mathbb{Q}}$. Take $X \in \mathcal{C}$ with $\operatorname{dim} X=d$. For $\Gamma \in \mathcal{C}_{\overline{\mathbb{Q}}}$ with $\operatorname{dim} \Gamma=1$ and $S \in \mathcal{C}$ and $\xi \in \mathrm{CH}^{r}(\Gamma / \mathbb{C} \times S \times X ; \mathbb{Q})$, consider the cycle induced map

$$
[\xi]_{*}: H^{1}(\Gamma / \mathbb{C}, \mathbb{Q}) \otimes H^{2 d_{S}-\nu+1}(S, \mathbb{Q}) \rightarrow H^{2 r-\nu}(X, \mathbb{Q}) .
$$

where $d_{S}=\operatorname{dim} S$. It is easy to see that

$$
[\xi]_{*}\left(H^{1}(\Gamma / \mathbb{C}, \mathbb{Q}) \otimes H^{2 d_{S}-\nu+1}(S, \mathbb{Q})\right) \subset H^{2 r-\nu}(X, \mathbb{Q}) \cap F^{r-\nu} H^{2 r-\nu}(X, \mathbb{C}) .
$$

Now introduce:

Definition 7.1. Write $H^{r-\nu, r}(X)=H^{r}\left(X, \Omega_{X / \mathbb{C}}^{r-\nu}\right)$. 
(i) Define $H_{\overline{\mathbb{Q}}}^{r-\nu, r}(X) \subset H^{r-\nu, r}(X)$ as the $\mathbb{C}$-vector space generated by the Hodge projected images of $[\xi]_{*}$ in 7.0, over all $\Gamma \in \mathcal{C}_{\overline{\mathbb{Q}}}$ of dimension 1 , and all $S \in \mathcal{C}$, and all $\xi \in C H^{r}(\Gamma / \mathbb{C} \times S \times X ; \mathbb{Q})$.

(ii) For $X_{o} \in \mathcal{C}_{\overline{\mathbb{Q}}}$, we introduce a variant $H_{\overline{\mathbb{Q}}}^{r-\nu, r}\left(X_{o} / \overline{\mathbb{Q}}\right) \subset H^{r-\nu, r}(X)\left(X=X_{o} \times{ }_{\overline{\mathbb{Q}}} \mathbb{C}\right)$ of the above definition, where we only consider $S \in \mathcal{C}_{\overline{\mathbb{Q}}}$ and where we allow $\xi \in C H^{r}((\Gamma \times S \times X) / \mathbb{C} ; \mathbb{Q})$.

For $X \in \mathcal{C}$ we recall the maps (see (3.7.2) and (3.7.3))

$$
\phi_{X, D R}^{r, \nu}: G r_{F_{B}}^{\nu} \mathrm{CH}^{r}(X ; \mathbb{Q}) \rightarrow \nabla D R^{r, \nu}(X) \quad \text { and } \quad \phi_{X}^{r, \nu}: G r_{F_{B}}^{\nu} \mathrm{CH}^{r}(X ; \mathbb{Q}) \rightarrow \nabla J^{r, \nu}(X) .
$$

The main result is the following:

Theorem 7.2. Assume $C(X)(c f . \S 1(v))$.

(i) If $\nu \geq 2$ and if $H_{\overline{\mathbb{Q}}}^{r-\nu, r}(X) \neq 0$, then there are an uncountable number of classes in $\operatorname{ker}\left(\phi_{X, D R}^{r, \nu}\right)$.

(ii) Let $X=X_{o} \times_{\overline{\mathbb{Q}}} \mathbb{C}$ with $X_{o} \in \mathcal{C}_{\overline{\mathbb{Q}}}$. If $\nu \geq 2$ and if $H_{\overline{\mathbb{Q}}}^{r-\nu, r}\left(X_{o} / \overline{\mathbb{Q}}\right) \neq 0$, then there are an uncountable number of classes in $\operatorname{ker}\left(\phi_{X}^{r, \nu}\right)$.

(iii) Assume Conjecture 6.4 is true. If $\nu \geq 2$ and if $H_{\overline{\mathbb{Q}}}^{r-\nu, r}(X) \neq 0$, then there are an uncountable number of classes in $\operatorname{ker}\left(\phi_{X}^{r, \nu}\right)$.

Proof of Theorem. Since the proofs of (ii) and (iii) are similar to and simpler than (i), we will just prove (i). By assumption, one can find $\Gamma / \overline{\mathbb{Q}}, S$ and a cycle $\xi \in \mathrm{CH}^{r}(\Gamma / \mathbb{C} \times S \times X)$ such that the map

$$
\xi_{*}: H^{1}(\Gamma / \mathbb{C}, \mathbb{C}) \rightarrow H^{\nu-1}(S, \mathbb{C}) \otimes\left(H^{2 r-\nu}(X, \mathbb{C}) / F^{r-\nu+1} H^{2 r-\nu}(X, \mathbb{C})\right),
$$

is nonzero. Note that $\nu \geq 2$ implies $\operatorname{dim} S \geq 1$, which will be exploited later.

In what follows, for a $\mathbb{Z}$ - or $\mathbb{Q}$-module $H$ of finite rank endowed with a mixed Hodge structure, we denote

$$
J(H)=W_{0} H \otimes \mathbb{C} /\left(F^{0} W_{0} H \otimes \mathbb{C}+W_{0} H\right) .
$$

It is naturally endowed with a structure of a complex Lie group and for a morphism $H \rightarrow H^{\prime}$, the induced map $J(H) \rightarrow J\left(H^{\prime}\right)$ is a homomorphism of complex Lie groups. We note that

$$
J^{r}(S \times X)=J\left(H^{2 r-1}(S \times X, \mathbb{Z})(r)\right)
$$

and the canonical isomorphism due to Carlson for a $\mathbb{Q}$-mixed Hodge structure $H$

$$
\operatorname{Ext}_{\mathrm{MHS}}^{1}(\mathbb{Q}(0), H) \simeq J(H) .
$$

Consider the composite map

$$
\Phi_{\xi}: \mathrm{CH}_{\operatorname{deg} 0}^{1}(\Gamma / \overline{\mathbb{Q}}) \rightarrow J^{1}(\Gamma / \mathbb{C}) \stackrel{\xi_{*}}{\longrightarrow} J^{r}(S \times X) \rightarrow J\left(H^{\nu-1}(S, \mathbb{Z}) \otimes H^{2 r-\nu}(X, \mathbb{Z})(r)\right),
$$

where the last map is induced by the projection to the Künneth component. For dense (Zariski) open $U \subset S$ let

$$
\Phi_{\xi, U}: \mathrm{CH}_{\operatorname{deg} 0}^{1}(\Gamma / \overline{\mathbb{Q}}) \rightarrow J\left(H^{\nu-1}(U, \mathbb{Z}) \otimes H^{2 r-\nu}(X, \mathbb{Z})(r)\right)
$$

be the composite of $\Phi_{\xi}$ with the natural map

$$
J\left(H^{\nu-1}(S, \mathbb{Z}) \otimes H^{2 r-\nu}(X, \mathbb{Z})(r)\right) \rightarrow J\left(H^{\nu-1}(U, \mathbb{Z}) \otimes H^{2 r-\nu}(X, \mathbb{Z})(r)\right) .
$$

A key to the proof of Theorem 7.2 is the following: 
Lemma 7.3. Under the above assumption,

$$
\lim _{U \vec{U} S} \operatorname{Image}\left(\Phi_{\xi, U}\right),
$$

has infinite rank.

We now finish the proof of Theorem 7.2 assuming Lemma 7.3. We choose spreads of $X$ and $S$ : Choose $M$ smooth over $\overline{\mathbb{Q}}$ and proper smooth morphisms $\mathcal{X} \rightarrow M$ and $\mathcal{S} \rightarrow M$ such that for a suitable $\eta:$ Spec $(\mathbb{C}) \rightarrow M$ whose image is the generic point, we have $\mathcal{X} \times{ }_{M} \operatorname{Spec}(\mathbb{C}) \simeq X$ and $\mathcal{S} \times{ }_{M} \operatorname{Spec}(\mathbb{C}) \simeq S$ by the base change via $\eta$. Correspondingly we have $\tilde{\xi} \in \mathrm{CH}^{r}\left(\Gamma \times \mathcal{X} \times{ }_{M} \mathcal{S} ; \mathbb{Q}\right)$ whose image under

$$
\eta^{*}: \mathrm{CH}^{r}\left(\Gamma \times \mathcal{X} \times{ }_{M} \mathcal{S} ; \mathbb{Q}\right) \rightarrow \mathrm{CH}^{r}(\Gamma \times X \times S ; \mathbb{Q})
$$

coincides with $\xi$. By [Ja], 7.2 (see also [Sa2], 5-1) one can lift the the Künneth components of the cohomology classes of the diagonal class $\left[\Delta_{X}\right] \in H^{2 d}(X \times X, \mathbb{Q}$ ) (which are algebraic by $C(X))$ to

$$
\tilde{\Delta}_{\mathcal{X}}(i, j) \in \mathrm{CH}^{d}\left(\mathcal{X} \times_{M} \mathcal{X} ; \mathbb{Q}\right) / \bigcap_{\mu \geq 1} F_{B}^{\mu} \mathrm{CH}^{d}\left(\mathcal{X} \times_{M} \mathcal{X} / M ; \mathbb{Q}\right) \quad(i+j=2 d)
$$

such that their restrictions to the fiber $X_{s}$ for every $s: \operatorname{Spec}(\mathbb{C}) \rightarrow M$

$$
\tilde{\Delta}_{X_{s}}(i, j) \in \mathrm{CH}^{d}\left(X_{s} \times X_{s} ; \mathbb{Q}\right) / \bigcap_{\mu \geq 1} F_{B}^{\mu} \mathrm{CH}^{d}\left(X_{s} \times X_{s} ; \mathbb{Q}\right) \quad(i+j=2 d)
$$

give a Chow-Künneth decomposition of the diagonal. Then we can let $\tilde{\Delta}_{\mathcal{X}}(2 d-2 r+\nu, 2 r-\nu)$ act on $\tilde{\xi}$ (and replace $\tilde{\xi}$ by the result) without changing (7.2.1). This will ensure that $\tilde{\xi}$ induces

$$
\Phi_{\tilde{\xi}}: \mathrm{CH}_{\operatorname{deg} 0}^{1}(\Gamma / \overline{\mathbb{Q}}) \rightarrow F_{B}^{\nu} \mathrm{CH}^{r}\left(\mathcal{X} \times_{M} \mathcal{S} / \mathcal{S} ; \mathbb{Q}\right) \rightarrow G r_{F_{B}}^{\nu} \mathrm{CH}^{r}\left(\mathcal{X} \times_{M} \mathcal{S} / \mathcal{S} ; \mathbb{Q}\right)
$$

and its image is contained in $\mathrm{CH}_{\mathrm{hom}}^{r}\left(\mathcal{X} \times{ }_{M} \mathcal{S} ; \mathbb{Q}\right)$. By the construction in $\S 6$, this means

$$
\operatorname{Image}\left(\Phi_{\tilde{\xi}}\right) \subset \operatorname{ker}\left(G r_{F_{B}}^{\nu} \mathrm{CH}^{r}\left(\mathcal{X} \times_{M} \mathcal{S} / \mathcal{S} ; \mathbb{Q}\right) \stackrel{\phi_{\mathcal{X}}^{r, \nu} \times_{M} \mathcal{S} / \mathcal{S}, D R}{\longrightarrow} \nabla D R^{r, \nu}\left(\mathcal{X} \times_{M} \mathcal{S} / \mathcal{S}\right)\right)
$$

By construction, $\Phi_{\xi}$ coincides with the composite of $\Phi_{\tilde{\xi}}$ and the map (cf. (6.2))

$$
\begin{aligned}
F_{B}^{\nu} \mathrm{CH}^{r}\left(\mathcal{X} \times{ }_{M} \mathcal{S} / \mathcal{S} ; \mathbb{Q}\right) \cap \mathrm{CH}_{\mathrm{hom}}^{r}\left(\mathcal{X} \times{ }_{M} \mathcal{S} ; \mathbb{Q}\right) \stackrel{\epsilon_{\mathcal{X} \times{ }_{M} \mathcal{S} / \mathcal{S}}^{r, \nu}}{\longrightarrow} \Xi^{r, \nu}\left(\mathcal{X} \times{ }_{M} \mathcal{S} / \mathcal{S}\right) \\
:=\operatorname{Ext}_{\mathrm{MHS}}^{1}\left(\mathbb{Q}(0), H^{\nu-1}\left(\mathcal{S} / \mathbb{C}, R^{2 r-\nu} f_{*} \mathbb{Q}(r)\right)\right),
\end{aligned}
$$

where $f: \mathcal{X} \times_{M} \mathcal{S} \rightarrow \mathcal{S}$ is the projection, and the map

$$
\Xi^{r, \nu}\left(\mathcal{X} \times{ }_{M} \mathcal{S} / \mathcal{S}\right) \rightarrow \operatorname{Ext}_{\mathrm{MHS}}^{1}\left(\mathbb{Q}(0), H^{\nu-1}(S, \mathbb{Q}) \otimes H^{2 r-\nu}(X, \mathbb{Q})(r)\right)=J\left(H^{\nu-1}(S, \mathbb{Q}) \otimes H^{2 r-\nu}(X, \mathbb{Q})(r)\right)
$$

which is the restriction via $S \hookrightarrow \mathcal{S} / \mathbb{C}$ induced by $\eta$ (Recall that $\eta: \operatorname{Spec}(\mathbb{C}) \rightarrow M$ gives rise to $\eta_{\mathbb{C}}$ : $\operatorname{Spec}(\mathbb{C}) \rightarrow M / \mathbb{C}$ and $S \simeq \mathcal{S} / \mathbb{C} \times_{M / \mathbb{C}} \operatorname{Spec}(\mathbb{C})$ by the base change via $\left.\eta_{\mathbb{C}}\right)$. Noting that $\epsilon_{\mathcal{X} \times{ }_{M} \mathcal{S} / \mathcal{S}}^{r, \nu}$ annihilates $\left.F_{B}^{\nu+1} \mathrm{CH}^{r}\left(\mathcal{X} \times{ }_{M} \mathcal{S} / \mathcal{S} ; \mathbb{Q}\right)\right)$, Lemma 7.3 implies that the image of

$$
\mathrm{CH}_{\operatorname{deg} 0}^{1}(\Gamma / \overline{\mathbb{Q}}) \stackrel{\Phi_{\tilde{\xi}}}{\longrightarrow} G r_{F_{B}}^{\nu} \mathrm{CH}^{r}\left(\mathcal{X} \times_{M} \mathcal{S} / \mathcal{S} ; \mathbb{Q}\right) \stackrel{j^{*}}{\longrightarrow} G r_{F_{B}}^{\nu} \mathrm{CH}^{r}\left(\mathcal{X} \times_{M} \mathcal{U} / \mathcal{U} ; \mathbb{Q}\right)
$$


has infinite rank in the limit over any dense open $j: \mathcal{U} \hookrightarrow \mathcal{S}$. Identify a point $p \in S$ with a morphism $p: \operatorname{Spec}(\mathbb{C}) \rightarrow S$ and let $\tilde{p}: \operatorname{Spec}(\mathbb{C}) \rightarrow \mathcal{S}$ be the composite of $p$ and $S \rightarrow \mathcal{S}$ induced by $\eta$. Then the composite of $\tilde{p}$ and $\mathcal{S} \rightarrow M$ is $\eta$ so that we have $X \simeq \mathcal{X} \times{ }_{M} \mathcal{S} \times \mathcal{S}$ Spec(C) by the base change via $\tilde{p}$. If the image of $\tilde{p}$ is the generic point, it induces the map

$$
\tilde{p}^{*}: \lim _{\overrightarrow{\mathcal{U} \subset \mathcal{S}}} G r_{F_{B}}^{\nu} \mathrm{CH}^{r}\left(\mathcal{X} \times_{M} \mathcal{U} ; \mathbb{Q}\right) \rightarrow G r_{F_{B}}^{\nu} \mathrm{CH}^{r}(X ; \mathbb{Q}),
$$

where $\mathcal{U}$ ranges over the dense open subschemes of $\mathcal{S}$, and it is injective by [Sa3], Rem.(2-1)(3). Therefore the composite map

$$
\Phi_{\tilde{\xi}, p}: \mathrm{CH}_{\operatorname{deg} 0}^{1}(\Gamma / \overline{\mathbb{Q}}) \stackrel{\Phi_{\tilde{\xi}}}{\rightarrow} G r_{F_{B}}^{\nu} \mathrm{CH}^{r}\left(\mathcal{X} \times_{M} \mathcal{S} / \mathcal{S} ; \mathbb{Q}\right) \stackrel{\tilde{p}^{*}}{\longrightarrow} G r_{F_{B}}^{\nu} \mathrm{CH}^{r}(X ; \mathbb{Q})
$$

has the image of infinite rank and it is contained in $\operatorname{ker}\left(\phi_{X, D R}^{r, \nu}\right)$ by $(*)$ and Proposition 6.5. Noting that $\Phi_{\tilde{\xi}, p}$ coincides with

$$
\mathrm{CH}_{\operatorname{deg} 0}^{1}(\Gamma / \overline{\mathbb{Q}}) \rightarrow \mathrm{CH}_{\operatorname{deg} 0}^{1}(\Gamma / \overline{\mathbb{Q}}) \otimes \mathrm{CH}_{0}(S ; \mathbb{Q}) \stackrel{\xi^{*}}{\longrightarrow} G r_{F_{B}}^{\nu} \mathrm{CH}^{r}(X ; \mathbb{Q})
$$

where the first map sends $\alpha \in \mathrm{CH}_{\operatorname{deg} 0}^{1}(\Gamma / \overline{\mathbb{Q}})$ to $\alpha \otimes[p]$, we have thus shown that the image of

$$
\xi_{*}: \mathrm{CH}_{\operatorname{deg} 0}^{1}(\Gamma / \overline{\mathbb{Q}}) \otimes \mathrm{CH}_{0}(S ; \mathbb{Q}) \rightarrow G r_{F_{B}}^{\nu} \mathrm{CH}^{r}(X ; \mathbb{Q}),
$$

is nontrivial and lies in $\operatorname{ker}\left(\phi_{X, D R}^{r, \nu}\right)$. Thus for suitable $P-Q \in \mathrm{CH}_{\operatorname{deg} 0}^{1}(\Gamma / \overline{\mathbb{Q}})$, we have a nontrivial cycle induced map

$$
\mathrm{CH}_{0}(S ; \mathbb{Q}) \rightarrow \operatorname{ker}\left(\phi_{X, D R}^{r, \nu}\right) \subset G r_{F_{B}}^{\nu} \mathrm{CH}^{r}(X ; \mathbb{Q}) .
$$

This induces a nontrivial composite map

$$
\lambda: S \rightarrow \mathrm{CH}_{0}(S / \mathbb{C} ; \mathbb{Q}) \rightarrow \operatorname{ker}\left(\phi_{X, D R}^{r, \nu}\right) \hookrightarrow G r_{F_{B}}^{\nu} \mathrm{CH}^{r}(X ; \mathbb{Q}),
$$

whose image by an easy argument using $\operatorname{dim} S \geq 1$, is more than a point. The standard arguments in the theory of Chow varieties imply that the fibers of $\lambda$ are $c$-closed (countably closed). I.e. the fibers of $\lambda$ are countable unions of proper subvarieties of $S$. This is the import of the theory in [R1-2], (also cf. [Sch1]), and it basically hinges on Lemma 7.4 below. Thus if the image of $\lambda$ were countable, then $S$ would be a countable union of proper subvarieties. This is impossible by Baire's Theorem, which completes the proof of Theorem 7.2 .

Lemma 7.4. Let $X \in \mathcal{C}$ and $Y$ be a projective variety over $\mathbb{C}$, and assume given a cycle induced map

$$
\kappa: Y \rightarrow C H^{r}(X ; \mathbb{Q}) .
$$

Then $\kappa^{-1}\left(F_{B}^{\nu} C H^{r}(X ; \mathbb{Q})\right)$ is a c-closed subset of $Y$. Furthermore, if $\kappa(Y) \subset F_{B}^{\nu} C H^{r}(X ; \mathbb{Q})$, then the fibers of the induced map $Y \rightarrow G r_{F_{B}}^{\nu} C H^{r}(X ; \mathbb{Q})$ are c-closed.

Proof. Omitted.

Now we prove Lemma 7.3.

Sublemma 7.3.1. Consider the map

$$
\xi_{*}: H^{1}(\Gamma / \mathbb{C}, \mathbb{Q}) \rightarrow H^{\nu-1}(S, \mathbb{Q}) \otimes H^{2 r-\nu}(X, \mathbb{Q})
$$

induced by $\xi$ (as in eqn. (7.2.1)). Then its image is not contained in

$$
N_{H}^{1} H^{\nu-1}(S, \mathbb{Q}) \otimes H^{2 r-\nu}(X, \mathbb{Q})+H^{\nu-1}(S, \mathbb{Q}) \otimes N_{H}^{r-\nu+1} H^{2 r-\nu}(X, \mathbb{Q}),
$$

where we recall $N_{H}^{p} H^{\bullet}(X, \mathbb{Q})$ is the largest subHodge structure of $H^{\bullet}(X, \mathbb{Q})$ lying in $F^{p} H^{\bullet}(X, \mathbb{C}) \cap H^{\bullet}(X, \mathbb{Q})$.

Proof of Claim. Obvious. 
Sublemma 7.3.2. The image of

$$
\left.\left.J^{1}(\Gamma / \mathbb{C}) \stackrel{\xi_{*}}{\rightarrow} J^{r}(S \times X) \rightarrow J\left(H^{\nu-1}(S, \mathbb{Z}) \otimes H^{2 r-\nu}(X, \mathbb{Z})(r)\right)\right) \rightarrow J\left(H^{\nu-1}(U, \mathbb{Z}) \otimes H^{2 r-\nu}(X, \mathbb{Z})(r)\right)\right)
$$

is nonzero.

Proof. Recall that

$$
J\left(H^{\nu-1}(S, \mathbb{Z}) \otimes H^{2 r-\nu}(X, \mathbb{Z})(r)\right)=V / L
$$

with

$$
V:=\left(H^{\nu-1}(S, \mathbb{C}) \otimes H^{2 r-\nu}(X, \mathbb{C})\right) / F^{r}\left(H^{\nu-1}(S, \mathbb{C}) \otimes H^{2 r-\nu}(X, \mathbb{C})\right)
$$

and $L \subset V$ a lattice. We observe that

$$
F^{r}\left(H^{\nu-1}(S, \mathbb{C}) \otimes H^{2 r-\nu}(X, \mathbb{C})\right) \subset H^{\nu-1}(S, \mathbb{C}) \otimes F^{r-\nu+1} H^{2 r-\nu}(X, \mathbb{C}) .
$$

By the assumption (cf. (7.2.1)) the image of $\left.J^{1}(\Gamma / \mathbb{C}) \stackrel{\xi_{*}}{\rightarrow} J^{r}(S \times X) \rightarrow J\left(H^{\nu-1}(S, \mathbb{Z}) \otimes H^{2 r-\nu}(X, \mathbb{Z})(r)\right)\right)$ is nonzero. By Sublemma 7.3.1 it now suffices to show that the kernel of

$$
J\left(H^{\nu-1}(S, \mathbb{Z}) \otimes H^{2 r-\nu}(X, \mathbb{Z})(r)\right) \rightarrow J\left(H^{\nu-1}(U, \mathbb{Z}) \otimes H^{2 r-\nu}(X, \mathbb{Z})(r)\right) .
$$

is contained in the image of

$$
J\left(N_{H}^{1} H^{\nu-1}(S, \mathbb{Q}) \otimes H^{2 r-\nu}(X, \mathbb{Q})\right)+J\left(H^{\nu-1}(S, \mathbb{Q}) \otimes N_{H}^{r+1-\nu} H^{2 r-\nu}(X, \mathbb{Q})\right) .
$$

Indeed one has a surjective map

$$
H^{\nu-1}(S, \mathbb{Q}) \otimes H^{2 r-\nu}(X, \mathbb{Q})(r) \rightarrow W_{\nu-1} H^{\nu-1}(U, \mathbb{Q}) \otimes H^{2 r-\nu}(X, \mathbb{Q})(r),
$$

whose kernel is contained in $N_{H}^{1} H^{\nu-1}(S, \mathbb{Q}) \otimes H^{2 r-\nu}(X, \mathbb{Q})(r)$. Correspondingly the kernel of

$$
J\left(H^{\nu-1}(S, \mathbb{Q}) \otimes H^{2 r-\nu}(X, \mathbb{Q})(r)\right) \rightarrow J\left(W_{\nu-1} H^{\nu-1}(U, \mathbb{Q}) \otimes H^{2 r-\nu}(X, \mathbb{Q})(r)\right)
$$

is contained in the image of $J\left(N_{H}^{1} H^{\nu-1}(S, \mathbb{Q}) \otimes H^{2 r-\nu}(X, \mathbb{Q})\right)$. Next, there is a short exact sequence

$$
\begin{gathered}
0 \rightarrow W_{\nu-1} H^{\nu-1}(U, \mathbb{Q}) \otimes H^{2 r-\nu}(X, \mathbb{Q})(r) \rightarrow W_{0}\left(H^{\nu-1}(U, \mathbb{Q}) \otimes H^{2 r-\nu}(X, \mathbb{Q})(r)\right) \\
\rightarrow G r_{W}^{0}\left(H^{\nu-1}(U, \mathbb{Q}) \otimes H^{2 r-\nu}(X, \mathbb{Q})(r)\right) \rightarrow 0 .
\end{gathered}
$$

Taking global Ext ${ }_{\mathrm{MHS}}$, it gives us the exact sequence

$$
\begin{gathered}
\operatorname{hom}_{\mathrm{MHS}}\left(\mathbb{Q}(0), G r_{W}^{0}\left(H^{\nu-1}(U, \mathbb{Q}) \otimes H^{2 r-\nu}(X, \mathbb{Q})(r)\right)\right) \rightarrow \\
J\left(W_{\nu-1} H^{\nu-1}(U, \mathbb{Q}) \otimes H^{2 r-\nu}(X, \mathbb{Q})(r)\right) \rightarrow J\left(H^{\nu-1}(U, \mathbb{Q}) \otimes H^{2 r-\nu}(X, \mathbb{Q})(r)\right) .
\end{gathered}
$$

Hence it suffices to show that the image of the first map is contained in

$$
J\left(W_{\nu-1} H^{\nu-1}(U, \mathbb{Q}) \otimes N_{H}^{r+1-\nu} H^{2 r-\nu}(X, \mathbb{Q})(r)\right) .
$$

We have a short exact sequence

$$
\begin{gathered}
0 \rightarrow W_{\nu-1} H^{\nu-1}(U, \mathbb{Q}) \otimes N_{H}^{r+1-\nu} H^{2 r-1}(X, \mathbb{Q})(r) \rightarrow W_{0}\left(H^{\nu-1}(U, \mathbb{Q}) \otimes N_{H}^{r+1-\nu} H^{2 r-1}(X, \mathbb{Q})(r)\right) \\
\rightarrow G r_{W}^{0}\left(H^{\nu-1}(U, \mathbb{Q}) \otimes N_{H}^{r+1-\nu} H^{2 r-\nu}(X, \mathbb{Q})(r)\right) \rightarrow 0 .
\end{gathered}
$$

Moreover, by using the fact $F^{p} H^{\nu-1}(U, \mathbb{C}) / F^{p+1}=0$ for $p>\nu-1$, one sees that the natural inclusion:

$$
\operatorname{hom}_{\mathrm{MHS}}\left(\mathbb{Q}(0), G r_{W}^{0}\left(H^{\nu-1}(U, \mathbb{Q}) \otimes N_{H}^{r+1-\nu} H^{2 r-\nu}(X, \mathbb{Q})(r)\right)\right) \quad \subset \quad \operatorname{hom}_{\mathrm{MHS}}\left(\mathbb{Q}(0), G r_{W}^{0}\left(H^{\nu-1}(U, \mathbb{Q}) \otimes H^{2 r-\nu}(X, \mathbb{Q})(r)\right)\right),
$$

is an equality. Finally the desired assertion follows from the commutative diagram:

$$
\begin{array}{ccc}
\operatorname{hom}_{\mathrm{MHS}}\left(\mathbb{Q}(0), G r_{W}^{0}\left(H^{\nu-1}(U, \mathbb{Q}) \otimes N_{H}^{r+1-\nu} H^{2 r-\nu}(X, \mathbb{Q})(r)\right)\right) & \rightarrow & J\left(W_{\nu-1} H^{\nu-1}(U, \mathbb{Q}) \otimes N_{H}^{r+1-\nu} H^{2 r-\nu}(X, \mathbb{Q})(r)\right) \\
\| & & \downarrow \\
\operatorname{hom}_{\mathrm{MHS}}\left(\mathbb{Q}(0), G r_{W}^{0}\left(H^{\nu-1}(U, \mathbb{Q}) \otimes H^{2 r-\nu}(X, \mathbb{Q})(r)\right)\right) & \rightarrow & J\left(W_{\nu-1} H^{\nu-1}(U, \mathbb{Q}) \otimes H^{2 r-\nu}(X, \mathbb{Q})(r)\right)
\end{array}
$$

In order to complete the proof of Lemma 7.3 we need the following key result. 
Lemma 7.5. Let $W$ be an Abelian variety over $\overline{\mathbb{Q}}$, and suppose that $W / \mathbb{C}$ contains an Abelian subvariety $V$ over $\mathbb{C}$. Then $W$ contains an Abelian subvariety over $\overline{\mathbb{Q}}$ of dimension $=\operatorname{dim} V$.

Proof. Construct a $\overline{\mathbb{Q}}$-spread

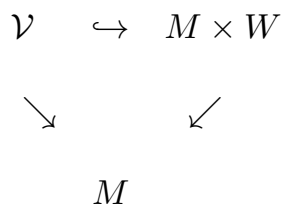

where $M, \mathcal{V}$ are defined over $\overline{\mathbb{Q}}$ and there is $\eta: \operatorname{Spec}(\mathbb{C}) \rightarrow M$ whose image is the generic point of $M$, such that $\mathcal{V} \times_{M} \operatorname{Spec}(\mathbb{C}) \simeq V$. Let $s \in M(\overline{\mathbb{Q}})$ be a general choice of closed point. Then the desired Abelian subvariety of $W$ is given by $\mathcal{V}_{s}$.

The following is probably well-known, but is an immediate consequence of Lemma 7.5.

Corollary 7.6. $W / \overline{\mathbb{Q}}$ is simple $\Leftrightarrow W \times_{\overline{\mathbb{Q}}} \mathbb{C}$ is simple.

Now we complete the proof of Lemma 7.3. Notice that $\mathrm{CH}_{\operatorname{deg} 0}^{1}(\Gamma / \overline{\mathbb{Q}})$ has the structure of an Abelian variety over $\overline{\mathbb{Q}}$. By Sublemma 7.3.2 and Corollary 7.6 together with Poincaré's complete reducibility theorem, there exists a simple Abelian subvariety $A / \overline{\mathbb{Q}} \subset \mathrm{CH}_{\operatorname{deg} 0}^{1}(\Gamma / \overline{\mathbb{Q}})$, for which the composite

$$
A / \mathbb{C} \hookrightarrow \mathrm{CH}_{\operatorname{deg} 0}^{1}(\Gamma / \mathbb{C}) \simeq J^{1}(\Gamma / \mathbb{C}) \rightarrow J\left(H^{\nu-1}(U, \mathbb{Z}) \otimes H^{2 r-\nu}(X, \mathbb{Z})(r)\right)
$$

is an isogeny onto its image. Now Lemma 7.3 follows from the fact that $A(\overline{\mathbb{Q}})$ has infinite rank ([F-G]).

Example 7.7. Let $X=C \times C$, where $C$ is a smooth projective curve defined over $\overline{\mathbb{Q}}$. In this case $C(X)$ trivially holds. Let

$$
\left[\Delta_{X}\right]=\sum_{p+q=4}\left[\Delta_{X}(p, q)\right] \in \bigoplus_{p+q=4} H^{2}(X \times X, \mathbb{Q}) \quad \text { with } \Delta_{X}(p, q) \in \mathrm{CH}^{2}(X \times X ; \mathbb{Q}),
$$

be the Künneth decomposition of the diagonal $X \hookrightarrow X \times X$. Using the notation in Theorem 7.2, we take $\Gamma=S=C$ and $r=\nu=2$ and $\xi=\Delta_{X}(2,2)$. Let $\eta$ be the generic point of $C$ and take $P, Q \in C(\overline{\mathbb{Q}})$. Noting

$$
\Delta_{X, *}=\Delta_{X}(0,4)_{*}+\Delta_{X}(1,3)_{*}+\Delta_{X}(2,2)_{*} \quad \text { on } \mathrm{CH}^{2}(X ; \mathbb{Q}),
$$

we get

$$
\xi_{*}((P-Q) \times \eta)=(P-Q) \times \eta-\Delta_{X}(1,3)_{*}((P-Q) \times \eta) .
$$

Next, we expand $\Delta_{X}(1,3)$ in terms of the diagonal $\Delta_{C}$ of $C \hookrightarrow C \times C$. Fix a rational point $O \in C(\overline{\mathbb{Q}})$. Let $T: C \times C \times C \times C \rightarrow C \times C \times C \times C$ be the map given by $T\left(t_{1}, t_{2}, t_{3}, t_{4}\right)=\left(t_{1}, t_{3}, t_{2}, t_{4}\right)$. Then

$$
\begin{gathered}
\Delta_{X}(1,3)_{*}((P-Q) \times \eta)=\left[T^{*}\left(C \times O \times \Delta_{C}+\Delta_{C} \times C \times O\right)\right]_{*}((P-Q) \times \eta) \\
=\left[T^{*}\left(\Delta_{C} \times C \times O\right)\right]_{*}((P-Q) \times \eta)=(P-Q) \times O .
\end{gathered}
$$

Thus we get

$$
\xi_{*}((P-Q) \times \eta)=(P-Q) \times(\eta-O),
$$

which is the cycle that was studied earlier by A. Rosenschoen and M. Saito [RS], and later by Matt Kerr [Ke1]. It should be pointed out that their results about a given 0 -cycle satisfying a certain condition, are more specific than what is presented here. 


\section{§8. A Class of examples with trivial Mumford-Griffiths invariant}

We first recall that if $V, W \in \mathcal{C}_{k}$, then $([\mathrm{K} 1])$

$$
B(V)+B(W) \Rightarrow B(V \times W) .
$$

Another fact that we use is the following. Let $m=\operatorname{dim} W$, and assume that $j: V \hookrightarrow W$ is of dimension $\ell$, being the cutout of $W$ by $m-\ell$ hyperplane sections. By the Lefschetz theorem, $j^{*}: H^{\ell}(W) \hookrightarrow H^{\ell}(V)$ is injective. Then $B(W) \Rightarrow B(V)$ and the surjective left inverse

$$
\left(j^{*}\right)^{-1}: H^{\ell}(V) \rightarrow H^{\ell}(W)
$$

is cycle induced. Indeed, one checks that

$$
j_{*} \circ j^{*}=L_{W}^{m-\ell}: H^{\ell}(W) \stackrel{\sim}{\rightarrow} H^{2 m-\ell}(W),
$$

where $L_{W}$ is the cup product with the polarizing class. If $\Lambda_{W}^{m-\ell}$ is the inverse to $L_{W}^{m-\ell}$, then we obtain $\left(j^{*}\right)^{-1}=\Lambda_{W}^{m-\ell} \circ j_{*}$ from the formula

$$
\left(j^{*}\right)^{-1} \circ j^{*}=\Lambda_{W}^{m-\ell} \circ j_{*} \circ j^{*}=\Lambda_{W}^{m-\ell} \circ L_{W}^{m-\ell}=\text { Identity }
$$

The following diagram makes the above construction transparent:

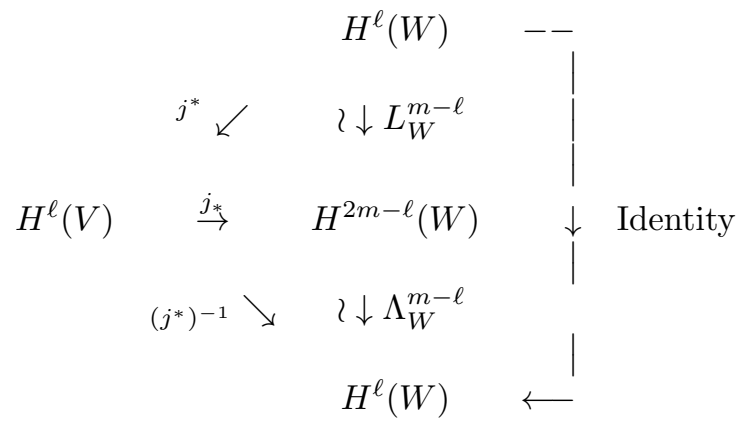

Lemma 8.1. (i) Let $X, Y \in \mathcal{C}$ and $\Gamma \in \mathcal{C}_{\overline{\mathbb{Q}}}$ with $\operatorname{dim} X=d, \operatorname{dim} \Gamma=1, \operatorname{dim} Y=d-1$. Assume that $B(Y)$ and $C(X)$ hold. Further, suppose that we are given a dominating morphism

$$
\lambda: \Gamma_{\mathbb{C}} \times Y \rightarrow X
$$

such that the induced map

$$
H^{0,1}(\Gamma / \mathbb{C}) \otimes H^{0, \nu-1}(Y) \rightarrow H^{0, \nu}(X),
$$

is nonzero for some integer $\nu \geq 2$. Then $H_{\mathbb{Q}}^{r-\nu, r}(X) \neq 0$ for $\nu \leq r \leq d$.

(ii) Further, if $X=X_{o} \times \overline{\mathbb{Q}} \mathbb{C}$ and $Y=Y_{o} \times \overline{\mathbb{Q}} \mathbb{C}$ with $X_{o}, Y_{o} \in \mathcal{C}_{\overline{\mathbb{Q}}}$, then $H_{\overline{\mathbb{Q}}}^{r-\nu, r}\left(X_{o} / \mathbb{Q}\right) \neq 0$ for $\nu \leq r \leq d$.

Proof. We only prove (i). The proof of (ii) is similar. The hard Lefschetz isomorphism implies that

$$
L_{X}^{r-\nu}: H^{0, \nu}(X) \hookrightarrow H^{r-\nu, r}(X),
$$


is injective. Now let $S$ be a smooth cutout of $Y$ by $d-\nu$ hyperplane sections, where $\operatorname{dim} S=\nu-1$. By what we said in the beginning of this section, there is a nontrivial cycle induced map

$$
H^{0,1}(\Gamma / \mathbb{C}) \otimes H^{0, \nu-1}(S) \rightarrow H^{r-\nu, r}(X)
$$

More explicitly, by the above discussion, the cycle inducing this map is given as follows: Let $\Delta_{\Gamma}$ be the diagonal of $\Gamma$ in $\Gamma \times \Gamma$. If $j: S \hookrightarrow Y$ is the inclusion, and if we identify a map $T_{*}$ with its associated correspondence $\left\{T_{*}\right\}$, then the cycle is

$$
\xi:=L_{X}^{r-\nu} \circ\left\{\lambda_{*}\right\} \circ\left(\left\langle\operatorname{Pr}_{13}^{*}\left(\Delta_{\Gamma_{\mathbb{C}}}\right) \bullet \operatorname{Pr}_{24}^{*}\left(\Lambda_{Y}^{d-\nu} \circ\left\{j_{*}\right\}\right)\right\rangle_{\Gamma \times S \times \Gamma \times Y}\right) \in \mathrm{CH}^{r}(\Gamma / \mathbb{C} \times S \times X ; \mathbb{Q}) .
$$

This gives a nonzero element of $H_{\mathbb{Q}}^{r-\nu, r}(X)$.

By Theorem 7.2, we arrive at the following:

Corollary 8.2. (i) Let the assumption be as in Lemma 8.1(i). Then, for $\nu \leq r \leq d, G r_{F_{B}}^{\nu} C H^{r}(X ; \mathbb{Q})$ contains an uncountable number of classes with trivial de Rham invariant.

(ii) Let the assumption be as in Lemma 8.1(ii). Then, for $\nu \leq r \leq d, G r_{F_{B}}^{\nu} C H^{r}(X ; \mathbb{Q})$ contains an uncountable number of classes with trivial Mumford-Griffiths invariant.

Corollary 8.3. Let $X_{o} \in \mathcal{C}_{\overline{\mathbb{Q}}}$ of dimension $d$, and write $X=X_{o} \times_{\overline{\mathbb{Q}}} \mathbb{C}$. Assume given smooth curves $\Gamma_{1} / \overline{\mathbb{Q}}, \ldots, \Gamma_{d} / \overline{\mathbb{Q}}$ and a dominating morphism

$$
\Gamma_{1} \times \cdots \times \Gamma_{d} \rightarrow X_{o}
$$

Further assume that $C(X)$ holds. If $H^{0}\left(X, \Omega_{X / \overline{\mathbb{Q}}}^{\nu}\right) \neq 0$ for some $\nu \geq 2$, then for $\nu \leq r \leq d, G r_{F_{B}}^{\nu} C H^{r}(X ; \mathbb{Q})$ contains an uncountable number of classes with trivial Mumford-Griffiths invariant.

Since any Abelian variety is dominated by a product of curves, we arrive at the following:

Corollary 8.3. Let $X_{o}$ be an Abelian variety over $\overline{\mathbb{Q}}$, of dimension $d$ and write $X=X_{o} \times \overline{\mathbb{Q}} \mathbb{C}$. . Then for any $2 \leq \nu \leq r \leq d, \quad G r_{F_{B}}^{\nu} C H^{r}(X ; \mathbb{Q})$ contains an uncountable number of classes with trivial Mumford-Griffiths invariant.

One can also arrive at similar results for Fermat hypersurfaces, using the fact that they are dominated by products of Fermat curves by [KS]. 


\section{ApPEndix: A CONJECTURAL OVERVIEW}

We would like to convey our thoughts about the possibility of a map from the space of algebraic cocycles $\Lambda_{\text {alg }}^{r, \nu}(X / S)$ to the space of Mumford-Griffiths invariants $\nabla J^{r, \nu}(X / S) \otimes \mathbb{C}$, where $\rho: X \rightarrow S$ is a smooth proper morphism of smooth quasiprojective varieties over $\overline{\mathbb{Q}}$. In a nutshell, we believe that it ought to exist. We base our observations on the situation with intersection cohomology considerations, and the various conjectures in the literature that seem to imply this.

(I) The issue is whether there is a map from

$$
\Lambda^{r, \nu}(X / S):=\operatorname{hom}_{\mathrm{MHS}}\left(\mathbb{Q}(0), H^{\nu}\left(S, R^{2 r-\nu} \rho_{*} \mathbb{Q}(r)\right) \subset H^{\nu}\left(S, R^{2 r-\nu} \rho_{*} \mathbb{C}\right)\right.
$$

at least on the space of algebraic cocycles $\Lambda_{\text {alg }}^{r, \nu}(X / S)$, (which is Hodge conjecturally all of $\Lambda^{r, \nu}(X / S)$ ) to the space of Mumford-Griffiths invariants $\nabla J^{r, \nu}(X / S) \otimes \mathbb{C}$. As already explained in $\S 8$, if there is such a map, and if $S$ is affine, then such a map must be injective. This is because, for $S$ affine, the de Rham invariants $\nabla D R^{r, \nu}(X / S) \otimes \mathbb{C}$ compute $H^{\nu}\left(S, R^{2 r-\nu} \rho_{*} \mathbb{C}\right)$, and the inclusion of $\Lambda^{r, \nu}(X / S)$ in the de Rham invariants factors through the map to $\nabla J^{r, \nu}(X / S) \otimes \mathbb{C}$.

Whether or not there exists a map to the Mumford-Griffiths invariants, we can still say that for $S$ affine, there is always a map

$$
\nabla J_{\mathrm{alg}}^{r, \nu}(X / S) \rightarrow \Lambda_{\mathrm{alg}}^{r, \nu}(X / S) \subset \Lambda^{r, \nu}(X / S)
$$

where $\nabla J_{\text {alg }}^{r, \nu}(X / S)$ is the image of $F^{\nu} \mathrm{CH}^{r}(X / S ; \mathbb{Q})$ in $\nabla J^{r, \nu}(X / S)$. One simply deduces this from the commutative diagram below, where $\Phi^{r, \nu}$ is injective.

$$
\begin{array}{ccc}
F^{\nu} \mathrm{CH}^{r}(X / S ; \mathbb{Q}) & \stackrel{\lambda_{X / S}^{r, \nu}}{\longrightarrow} & \Lambda^{r, \nu}(X / S) \\
\downarrow & & \downarrow \Phi^{r, \nu} \\
\nabla J^{r, \nu}(X / S) & \rightarrow & \nabla D R^{r, \nu}(X / S) \otimes \mathbb{C}
\end{array}
$$

The question then is, in more precise terms, is the following.

Question A.0. For $S$ quasiprojective, does there exist a map going the other way, viz.,

$$
\Lambda_{\text {alg }}^{r, \nu}(X / S) \rightarrow \nabla J^{r, \nu}(X / U) \otimes \mathbb{C},
$$

where $U / \overline{\mathbb{Q}} \subset S$ is any affine open subvariety? [Note: If $S=U$ is affine, then this map must necessarily be injective, if it exists.]

(II) Enter intersection cohomology: We work with this diagram:

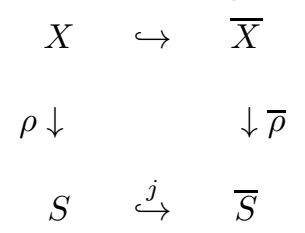

where $Y:=\bar{X} \backslash X, \Sigma:=\bar{S} \backslash S$ are NCD's, $\rho$ is smooth and proper, and $\bar{X}, \bar{S}$ are smooth projective over $\overline{\mathbb{Q}}$. We recall a conjecture of Zucker and Brylinski. First, let $\mathbb{V}=R^{m} \rho_{*} \mathbb{C}$ over $S$. Put

$$
\mathcal{G}^{i}=\left\{g \in \Omega \frac{i}{S}\langle\log \Sigma\rangle \otimes \overline{\mathcal{V}} \mid \operatorname{Res}_{\tilde{\Sigma}_{J}} g \in N_{J} V\right\},
$$

where $\overline{\mathcal{V}}$ is the privileged extension of the bundle associated to $\mathbb{V}$ over $S$ to $\bar{S}$ (see [B-Z] p. 63 for the definition of $\overline{\mathcal{V}}$ ), and $N_{J}$ for a multiindex $J$ corresponds to monodromy. (See (III) below for a definition of the MHS $V$.) All terminology in the conjecture below can be found in [B-Z]. Then according to [B-Z], "We suspect that the means are available to verify: 
Conjecture A.1. ([B-Z](3.19)) The complex $\mathcal{G} \bullet$ together with the [Hodge] filtration induced from (3-8) [of [B-Z]], completes [the middle perversity cochain complex] $\underline{\underline{I C}} \bullet\left(\bar{S}, \mathbb{V}_{A}\right)$ to a cohomological A-Hodge complex of weight $m$, such that the Hodge structure it gives on $I H^{i}(\bar{S}, \mathbb{V})$ coincides with that of (3.16) [of [B-Z]]."

For the benefit of the reader, we will clarify the statement of Conjecture A.1. First of all, we make use of the identification from $[\mathrm{C}-\mathrm{K}-\mathrm{S} 2], I H^{i}(\bar{S}, \mathbb{V}) \simeq H_{(2)}^{i}(S, \mathbb{V})$, where the former is intersection cohomology and the latter is $L^{2}$-cohomology, which has a pure Hodge structure by [C-K-S2]. This defines the Hodge structure on $I H^{i}(\bar{S}, \mathbb{V})$. (Alternatively, one has a Hodge structure on $I H^{i}(\bar{S}, \mathbb{V})$ from the work of M. Saito ([MSa1].) The privileged extension $\overline{\mathcal{V}}$ has corresponding Hodge filtration subbundles $\overline{\mathcal{F}}^{\bullet} \subset \overline{\mathcal{V}}$, which together with the monodromy weight filtration, determine a MHS on the central fiber $V$. One has a corresponding filtered subcomplex $\mathcal{F}^{p} \mathcal{G}^{i} \subset \mathcal{G}^{i}$, where we observe that $\mathcal{F}^{p} \mathcal{G}^{i} \subset \Omega \frac{i}{S}\langle\log \Sigma\rangle \otimes \overline{\mathcal{F}}^{p-i}$, where the latter sheaf is coherent. The import of Conjecture A.1 is that

$$
F^{\bullet} I H^{\bullet}(\bar{S}, \mathbb{V}) \simeq \mathbb{H}^{\bullet}\left(\mathcal{F}^{\bullet} \mathcal{G}^{\bullet}\right)
$$

Note that

$$
\left.\mathcal{F}^{p} \mathcal{G}^{i}\right|_{S}=\Omega_{S}^{i} \otimes F^{p-i} R^{m} \rho_{*} \mathbb{C}
$$

Thus an immediate consequence of this conjecture (with $m=2 r-\nu$ and $p=r-\nu$ ) is the existence of a map:

$$
\left\{H_{(2)}^{\nu}\left(S, R^{2 r-\nu} \rho_{*} \mathbb{C}\right)\right\}^{r, r} \rightarrow \nabla J^{r, \nu}(X / U) \otimes \mathbb{C}
$$

where again $U / \overline{\mathbb{Q}} \subset S$ is any affine open subvariety.

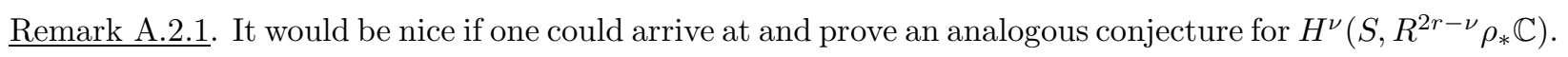

(III) Relating this to the original problem: We will now assume given our map in (A.2) above. Note that there is a natural map

$$
H_{(2)}^{\nu}\left(S, R^{2 r-\nu} \rho_{*} \mathbb{C}\right) \rightarrow H^{\nu}\left(S, R^{2 r-\nu} \rho_{*} \mathbb{C}\right) .
$$

Of course, in light of $[\mathrm{A}]$, one conjectures this to be a morphism of mixed Hodge structures. Using norm estimates on the weight filtration that arise from the work of [C-K-S1], one argues that:

Proposition A.3. There is a natural map

$$
H^{\nu}\left(\bar{S}, j_{*} R^{2 r-\nu} \rho_{*} \mathbb{C}\right) \rightarrow H_{(2)}^{\nu}\left(S, R^{2 r-\nu} \rho_{*} \mathbb{C}\right) .
$$

Proof. It suffices to construct a map:

$$
H^{\nu}\left(\bar{S}, j_{*} R^{2 r-\nu} \rho_{*} \mathbb{C}\right) \rightarrow I H^{\nu}\left(\bar{S}, R^{2 r-\nu} \rho_{*} \mathbb{C}\right) .
$$

Let $\underline{I C} \bullet(\bar{S}, \mathbb{V})$ be the middle perversity intersection cochain complex, where $\mathbb{V}=R^{2 r-\nu} \rho_{*} \mathbb{C}$. Then

$$
I H^{\nu}(\bar{S}, \mathbb{V}):=\mathbb{H}^{\nu}(\underline{I C} \cdot(\bar{S}, \mathbb{V}))
$$

The "first" hypercohomology spectral sequence ([G-H]) computing $\mathbb{H}^{\nu}(\underline{I C} \bullet(\bar{S}, \mathbb{V}))$ contains the $E_{2}$-term:

$$
{ }^{\prime} E_{2}^{\nu, 0}:=H^{\nu}\left(\bar{S}, \mathcal{H}^{0}(\underline{I C} \bullet(\bar{S}, \mathbb{V})) .\right.
$$


Therefore, if there is a natural map

$$
\tau: j_{*} \mathbb{V} \rightarrow \mathcal{H}^{0}(\underline{\underline{I C}} \cdot(\bar{S}, \mathbb{V}))
$$

this, together with partial degeneration, leads to maps:

$$
H^{\nu}\left(\bar{S}, j_{*} \mathbb{V}\right) \rightarrow{ }^{\prime} E_{2}^{\nu, 0} \rightarrow{ }^{\prime} E_{\infty}^{\nu, 0} \hookrightarrow I H^{\nu}(\bar{S}, \mathbb{V}),
$$

and hence Proposition A.3. To construct $\tau$, by passing to a finite cover of $S$, one can assume that the local monodromy transformations about the NCD $\Sigma \subset \bar{S}$ are unipotent. In a classical neighbourhood $U_{a n} \cap S \simeq\left(\Delta^{*}\right)^{m} \times \Delta^{\operatorname{dim} S-m}$. There is no loss of generality in deleting factors $\Delta$. Thus we may assume the local description $U_{a n} \cap S \simeq\left(\Delta^{*}\right)^{m}$. Let $T_{j}, j=1, \ldots, m$ be the local monodromy transformations, and put $N_{j}=\log T_{j}$. Let $\mathcal{V}=\mathcal{O}_{S}(\mathbb{V})$, and $\overline{\mathcal{V}}$ the canonical extension over $\Delta^{m}$. The stalk of $\overline{\mathcal{V}}$ over $0:=(0, \ldots 0) \in \Delta^{m}$ will be denoted by $V$. Put

$$
B^{p}\left(N_{1}, \ldots, N_{m} ; V\right)=\bigoplus_{1 \leq j_{1}<\cdots<j_{p} \leq m} N_{j_{1}} N_{j_{2}} \cdots N_{j_{p}} V .
$$

Define the differential

$$
(-1)^{s-1} N_{j_{s}}: N_{j_{1}} \cdots \widehat{N_{j_{s}}} \cdots N_{j_{p}} V \rightarrow N_{j_{1}} N_{j_{2}} \cdots N_{j_{p}} V
$$

on the various summands of $B^{p-1}\left(N_{1}, \ldots, N_{m} ; V\right)$. This turns $B^{\bullet}\left(N_{1}, \ldots, N_{m} ; V\right)$ into a complex. It is well known ([C-K-S2]) that

$$
H_{(2)}^{*}\left(U_{a n}, \mathbb{V}\right) \simeq H^{*}\left(B^{\bullet}\left(N_{1}, \ldots, N_{m} ; V\right)\right) \simeq I H^{*}\left(U_{a n}, \mathbb{V}\right)=\left(\mathcal{H}^{*}(\underline{I C} \cdot(\bar{S}, \mathbb{V}))\right)_{0} .
$$

The existence of $\tau$ follows by noticing that the invariant cycles include

$$
\left(j_{*} \mathbb{V}\right)_{0} \subset H^{0}\left(B^{\bullet}\left(N_{1}, \ldots, N_{m} ; V\right)\right) .
$$

Using the natural map $R^{2 r-\nu} \bar{\rho}_{*} \mathbb{C} \rightarrow j_{*} R^{2 r-\nu} \rho_{*} \mathbb{C}$, together with Proposition A.3, we deduce:

Corollary A.4. There is a natural map

$$
H^{\nu}\left(\bar{S}, R^{2 r-\nu} \bar{\rho}_{*} \mathbb{C}\right) \rightarrow H_{(2)}^{\nu}\left(S, R^{2 r-\nu} \rho_{*} \mathbb{C}\right) \simeq I H^{\nu}\left(\bar{S}, R^{2 r-\nu} \rho_{*} \mathbb{C}\right) .
$$

In light of the mixed Hodge structure results in $[\mathrm{A}]$, it is natural to expect the following.

Conjecture A.5. The map in A.4 is a morphism of MHS.

Notice that $H^{\nu}\left(\bar{S}, R^{2 r-\nu} \bar{\rho}_{*} \mathbb{C}\right)$ is the $E_{2}$ term of the Leray spectral sequence for $\bar{\rho}$.

Corollary A.6. Under the assumption of Conjecture A.5, there is an induced morphism of Hodge structures:

$$
G r_{\mathcal{L}}^{\nu} H^{2 r}(\bar{X}, \mathbb{C}) \rightarrow H_{(2)}^{\nu}\left(S, R^{2 r-\nu} \rho_{*} \mathbb{C}\right),
$$

where $G r_{\mathcal{L}}^{\nu} H^{2 r}(\bar{X}, \mathbb{C})$ is the graded Leray filtration.

Proof. To see this, observe that $H_{(2)}^{\nu}\left(S, R^{2 r-\nu} \rho_{*} \mathbb{C}\right)$ has a pure Hodge structure of weight $2 r$, and that by $[\mathrm{A}], H^{p}\left(\bar{S}, R^{q} \bar{\rho}_{*} \mathbb{C}\right)$ has a mixed Hodge structure of weight $\leq p+q$; moreover the differentials of the Leray spectral sequence are morphisms of MHS (see $[\mathrm{A}]$ ). Granted we accept Conjecture A.5, it follows that the composite

$$
H^{\nu-2}\left(\bar{S}, R^{2 r-\nu+1} \bar{\rho}_{*} \mathbb{C}\right) \stackrel{d_{2}}{\longrightarrow} H^{\nu}\left(\bar{S}, R^{2 r-\nu} \bar{\rho}_{*} \mathbb{C}\right) \rightarrow H_{(2)}^{\nu}\left(S, R^{2 r-\nu} \rho_{*} \mathbb{C}\right)
$$

is zero. Applying this same reasoning to the $d_{r}$ 's for $r \geq 3$ leads to Corollary A.6. 
Corollary A.7. Let us assume that Conjectures A.1 and A.5 hold. Then for $U / \overline{\mathbb{Q}} \subset S$ affine, there is a map

$$
\left\{G r_{\mathcal{L}}^{\nu} H^{2 r}(\bar{X}, \mathbb{C})\right\}^{r, r} \rightarrow \nabla J^{r, \nu}(X / U) \otimes \mathbb{C}
$$

It is natural then to ask whether one can replace $\bar{X}$ in $(*)$ by $X$ ? The answer appears to be yes, provided that one restricts to algebraic cocyles. The reason is this. Notice that by purity of Hodge structures, and polarization, $\operatorname{Gr}_{\mathcal{L}}^{\nu} H^{2 r}(\bar{X}, \mathbb{C}) \subset H^{2 r}(\bar{X}, \mathbb{C})$ as Hodge structures. Let's assume the Hodge conjecture for $Y$. More specifically, if we write the NCD $Y$ in the form $Y=\bigcup_{j} Y_{j}$, where $Y_{j}$ is smooth, then we are assuming the Hodge conjecture for each $Y_{j}$. Then the kernel of $H_{\mathrm{alg}}^{2 r}(\bar{X}) \rightarrow H^{2 r}(X)$ involves algebraic cocycles supported on $Y$. But any algebraic cycle supported on $Y$ goes to zero in $\mathrm{CH}^{r}(X)$. We want to make this more precise. We need the following.

Lemma A.8. Consider the composite of the natural maps

$$
\tau: F_{\mathcal{L}}^{\nu} H^{2 r}(\bar{X}, \mathbb{Q}) \rightarrow F_{\mathcal{L}}^{\nu} H^{2 r}(X, \mathbb{Q}) \rightarrow G r_{\mathcal{L}}^{\nu} H^{2 r}(X, \mathbb{Q}) \simeq H^{\nu}\left(S, R^{2 r-\nu} \rho_{*} \mathbb{Q}\right),
$$

where the first map is the restriction and the isomorphism comes from the degeneration of the Leray spectral sequence for $\rho: X \rightarrow S$. Note that it is a map of Hodge structures and hence induces the map

$$
G r_{\mathcal{L}}^{\nu} H^{2 r}(\bar{X}, \mathbb{Q}) \rightarrow W_{2 r} H^{\nu}\left(S, R^{2 r-\nu} \rho_{*} \mathbb{Q}\right)=G r_{W}^{2 r} H^{\nu}\left(S, R^{2 r-\nu} \rho_{*} \mathbb{Q}\right) .
$$

Then the last map is a surjective morphism of $H S$.

Proof. First of all, by $[\mathrm{A}]$, the weight of $H_{c}^{\nu}\left(S, R^{2 r-\nu} \rho_{*} \mathbb{Q}\right)$ is $\leq 2 r$. Hence by duality, the lowest weight space of $H^{\nu}\left(S, R^{2 r-\nu} \rho_{*} \mathbb{Q}\right)$ is $2 r$. That the above is a morphism of MHS is the import of [A]. This, together with cohomology with compact supports yields the exact sequence of MHS:

$$
H^{\nu-1}\left(\Sigma, R^{2 r-\nu} \bar{\rho}_{*} \mathbb{Q}\right) \rightarrow H_{c}^{\nu}\left(S, R^{2 r-\nu} \rho_{*} \mathbb{Q}\right) \rightarrow H^{\nu}\left(\bar{S}, R^{2 r-\nu} \bar{\rho}_{*} \mathbb{Q}\right) .
$$

Since $Y \rightarrow \Sigma$ is a morphism of projective varieties, the weight of $H^{\nu-1}\left(\Sigma, R^{2 r-\nu} \rho_{*} \mathbb{Q}\right)$ is $\leq 2 r-1$. Thus we have the inclusion

$$
G r_{W}^{2 r} H_{c}^{\nu}\left(S, R^{2 r-\nu} \rho_{*} \mathbb{Q}\right) \hookrightarrow G r_{W}^{2 r} H^{\nu}\left(\bar{S}, R^{2 r-\nu} \bar{\rho}_{*} \mathbb{Q}\right) .
$$

By compatibility of Leray spectral sequences together with a weight argument similar to the proof of Corollary A.6, this induces an injection:

$$
G r_{W}^{2 r} H_{c}^{\nu}\left(S, R^{2 r-\nu} \rho_{*} \mathbb{Q}\right) \hookrightarrow G r_{W}^{2 r} G r_{\mathcal{L}}^{\nu} H^{2 r}(\bar{X}, \mathbb{Q})=G r_{\mathcal{L}}^{\nu} H^{2 r}(\bar{X}, \mathbb{Q}) .
$$

The Lemma A.8 now follows from duality.

Corollary A.9. (i) For all $\nu \geq 0$, the kernel of $\tau$ in Lemma A.8 lies in the image

$$
\left\{H_{Y}^{2 r}(\bar{X}, \mathbb{Q})+F_{\mathcal{L}}^{\nu+1} H^{2 r}(\bar{X}, \mathbb{Q})\right\} \rightarrow H^{2 r}(\bar{X}, \mathbb{Q}) .
$$

(ii) For all $\nu \geq 0$, the natural map

$$
F_{\mathcal{L}}^{\nu} H^{2 r}(\bar{X}, \mathbb{Q}) \rightarrow W_{2 r} F_{\mathcal{L}}^{\nu} H^{2 r}(X, \mathbb{Q}),
$$

is a surjective morphism of $H S$.

Proof. Part (i): If $\xi \in F_{\mathcal{L}}^{\nu} H^{2 r}(\bar{X}, \mathbb{Q})$ restricts to a class in $F_{\mathcal{L}}^{\nu+1} H^{2 r}(X, \mathbb{Q})$, then by a repeated application of Lemma A.8, one can find $\xi^{\prime} \in F_{\mathcal{L}}^{\nu+1} H^{2 r}(\bar{X}, \mathbb{Q})$ such that $\xi-\xi^{\prime}$ restricts to zero in $H^{2 r}(X, \mathbb{Q})$. This implies part (i). The proof of part (ii) is again a repeated application of Lemma A.8, and will be left to the reader.

We now arrive at the following: 
Theorem A.10. Let us assume that Conjectures A.1 and A.5 hold, and that the Hodge conjecture holds. Then Question A.O is answered in the affirmative.

Proof. We have a commutative diagram

$$
\begin{array}{ccc}
\mathrm{CH}^{r}(\bar{X}) & \rightarrow & \mathrm{CH}^{r}(X) \\
\downarrow c_{\bar{X}}^{r} & & \downarrow c_{X}^{r} \\
H^{2 r}(\bar{X}, \mathbb{Q}(r)) & \rightarrow & H^{2 r}(X, \mathbb{Q}(r)) \\
\cup & & \bigcup \\
F_{\mathcal{L}}^{\nu} H^{2 r}(\bar{X}, \mathbb{Q}(r)) & \rightarrow & F_{\mathcal{L}}^{\nu} H^{2 r}(X, \mathbb{Q}(r))
\end{array}
$$

where $c_{X}^{r}$ and $c_{X}^{r}$ are the cycle class maps. One can show that $c_{X}^{r}\left(F_{B}^{\nu} \mathrm{CH}^{r}(X / S)\right) \subset F_{\mathcal{L}}^{\nu} H^{2 r}(X, \mathbb{Q}(r))$ by the same argument as [Sa3], Prop.(2-1) and that $\lambda_{X / S}^{r, \nu}$ is identified with the composite map:

$$
\psi: F_{B}^{\nu} \mathrm{CH}^{r}(X / S) \stackrel{c_{X}^{r}}{\longrightarrow} F_{\mathcal{L}}^{\nu} H^{2 r}(X, \mathbb{Q}(r)) \rightarrow H^{\nu}\left(S, R^{2 r-\nu} \rho_{*} \mathbb{Q}(r)\right) .
$$

Our aim is to construct a natural map Image $(\psi) \rightarrow \nabla J^{r, \nu}(X / S) \otimes \mathbb{C}$ by using the map $(*)$ in Corollary A.7. Put

$$
\mathrm{CH}^{r}(\bar{X}) \supset \Xi:=\left(c \frac{r}{X}\right)^{-1}\left(F_{\mathcal{L}}^{\nu} H^{2 r}(\bar{X}, \mathbb{Q}(r)) \cap H^{2 r}(\bar{X}, \mathbb{C})^{r, r}\right) \cap\left(j^{*}\right)^{-1}\left(F_{B}^{\nu} \mathrm{CH}^{r}(X / S)\right),
$$

where $j^{*}: \mathrm{CH}^{r}(\bar{X}) \rightarrow \mathrm{CH}^{r}(X)$ is the restriction. We have the following commutative diagrams:

$$
\begin{array}{ccc}
\Xi & \stackrel{j^{*}}{\longrightarrow} & F_{B}^{\nu} \mathrm{CH}^{r}(X / S) \\
\downarrow c_{\bar{X}}^{r} & \downarrow \psi \\
F_{\mathcal{L}}^{\nu} H^{2 r}(\bar{X}, \mathbb{Q}(r)) \cap H^{2 r}(\bar{X}, \mathbb{C})^{r, r} & \stackrel{\tau}{\rightarrow} & H^{\nu}\left(S, R^{2 r-\nu} \rho_{*} \mathbb{Q}(r)\right), \\
\Xi & \stackrel{j^{*}}{\longrightarrow} & F_{B}^{\nu} \mathrm{CH}^{r}(X / S) \\
\downarrow c_{\bar{X}}^{r} & & \downarrow \phi_{X / S}^{r, \nu} \\
F_{\mathcal{L}}^{\nu} H^{2 r}(\bar{X}, \mathbb{Q}(r)) \cap H^{2 r}(\bar{X}, \mathbb{C})^{r, r} & & \nabla J^{r, \nu}(X / S) \\
\downarrow & & \downarrow \\
\operatorname{Gr}_{\mathcal{L}}^{\nu} H^{2 r}(\bar{X}, \mathbb{C})^{r, r} & \stackrel{(*)}{\longrightarrow} & \nabla J^{r, \nu}(X / S) \otimes \mathbb{C},
\end{array}
$$

Assuming the Hodge conjecture for the irreducible components of $Y$, Corollary A.9 (i) implies that the kernel of $\tau$ in the former diagram is contained in the sum of $F_{\mathcal{L}}^{\nu+1} H^{2 r}(\bar{X}, \mathbb{Q}(r))$ and the image under $c_{\bar{X}}^{r}$ of the subgroup of $\mathrm{CH}^{r}(\bar{X})$ of the cycles supported on $Y$, which goes to zero under the restriction $j^{*}$. Hence, by a diagram chase and Hodge conjecture for $c_{\bar{X}}^{r}$, to construct a natural map Image $(\psi) \rightarrow \nabla J^{r, \nu}(X / S) \otimes \mathbb{C}$, it suffices to show the surjectivity of $\Xi \stackrel{j^{*}}{\rightarrow} F_{B}^{\nu} \mathrm{CH}^{r}(X / S)$. 
Let $\alpha \in F_{B}^{\nu} \mathrm{CH}^{r}(X / S)$, and consider its image $[\alpha]:=c_{X}^{r}(\alpha) \in W_{2 r} F_{\mathcal{L}}^{\nu} H^{2 r}(X, \mathbb{Q}(r))$. By Corollary A.9 (ii), there exists

$$
\overline{[\alpha]} \in F_{\mathcal{L}}^{\nu} H^{2 r}(\bar{X}, \mathbb{Q}(r)) \cap H^{2 r}(\bar{X}, \mathbb{C})^{r, r},
$$

which restricts to $[\alpha]$. Next $\alpha$ is the restriction of a class $\bar{\alpha} \in \mathrm{CH}^{r}(\bar{X} ; \mathbb{Q})$. Put $[\bar{\alpha}]=c_{\bar{X}}^{r}(\bar{\alpha})$. Thus

$$
[\bar{\alpha}]-\overline{[\alpha]} \in \operatorname{ker}\left(H^{2 r}(\bar{X}, \mathbb{Q}(r)) \rightarrow H^{2 r}(X, \mathbb{Q}(r))\right) \cap H^{2 r}(\bar{X}, \mathbb{C})^{r, r},
$$

and hence by the Hodge conjecture, $[\bar{\alpha}]-\overline{[\alpha]}=c_{\bar{X}}^{r}(\beta)$, where $\beta \in \mathrm{CH}^{r}(\bar{X})$ is an algebraic cycle supported on $Y$. Notice that

$$
\left.(\bar{\alpha}-\beta)\right|_{X}=\alpha \in F_{B}^{\nu} \mathrm{CH}^{r}(X / S),
$$

as any cycle supported on $Y$ goes to zero under the restriction $j^{*}: \mathrm{CH}^{r}(\bar{X}) \rightarrow \mathrm{CH}^{r}(X)$. Furthermore

$$
c_{\bar{X}}^{r}(\bar{\alpha}-\beta)=\overline{[\alpha]} \in F_{\mathcal{L}}^{\nu} H^{2 r}(\bar{X}, \mathbb{Q}(r)) \cap H^{2 r}(\bar{X}, \mathbb{C})^{r, r},
$$

which proves the desired assertion.

Recall from $\S 8$ that in the case where $S$ is affine, the composite

$$
\Lambda_{\mathrm{alg}}^{r, \nu}(X / S) \rightarrow \nabla J^{r, \nu}(X / S) \otimes \mathbb{C} \rightarrow \nabla D R^{r, \nu}(X / S) \otimes \mathbb{C}
$$

is injective. ${ }^{3}$ Thus the somewhat surprising conclusion from all of this, and from a conjectural point of view, is that with regard to space of algebraic cycles, one loses no information by passing from Mumford-Griffiths invariants to de Rham invariants. Thus in particular, this would enable us to replace classes with trivial de Rham invariant, by trivial Mumford-Griffiths invariant in sections 7 and 8.

Finally, in the case $\operatorname{dim} S=1$, Theorem A.10 holds without any conjectural assumption. By [Z], one has morphisms of MHS:

$$
G r_{\mathcal{L}}^{1} H^{2 r}(\bar{X}, \mathbb{C})=H^{1}\left(\bar{S}, R^{2 r-1} \bar{\rho}_{*} \mathbb{C}\right) \simeq H^{1}\left(\bar{S}, j_{*} R^{2 r-1} \rho_{*} \mathbb{C}\right) \hookrightarrow H^{1}\left(S, R^{2 r-1} \rho_{*} \mathbb{C}\right) .
$$

Note that $H^{2}\left(S, R^{2 r-2} \rho_{*} \mathbb{C}\right)=0$ if $S$ is affine.

\footnotetext{
${ }^{3}$ Warning: The natural map $\nabla J^{r, \nu}(X / S) \rightarrow \nabla D R^{r, \nu}(X / S)$ is not injective, as one can clearly see from the product case $X=X_{o} \times S$.
} 


\section{REFERENCES}

[A] D. Arapura, The Leray filtration is motivic. http://www.math.purdue.edu/ dvb/.

[As1] M. Asakura M., Motives and algebraic de Rham cohomology, in: The Arithmetic and Geometry of Algebraic Cycles, Proceedings of the CRM Summer School, June 7-19, 1998, Banff, Alberta, Canada (Editors: B. Gordon, J. Lewis, S. Müller-Stach, S. Saito and N. Yui), CRM Proceedings and Lecture Notes, 24 (2000), 133-155, AMS.

[As2] Arithmetic Hodge structure and nonvanishing of the cycle class of 0-cycles, $K$-theory, 27 (2002), 273-280.

[Bei] A. Beilinson, Notes on absolute Hodge cohomology; Applications of Algebraic $K$-Theory to Algebraic Geometry and Number Theory, Contemporary Mathematics 55 (I), American Mathematical Society, Providence (1986), 35-68.

[Bl] S. Bloch; Lectures on Algebraic Cycles, Duke Univ. Math. Series 4 (1980)

[Bo] C. Borcea, Deforming varieties of $k$-planes of projective complete intersections, Pacific Journal of Math. 143 (1990), 25-36, Sijthoff and Noordhoff.

[B-Z] J.-L. Brylinski and S. Zucker, An overview of recent advances in Hodge theory, In: Complex Manifolds, Springer-Verlag, New York, 1997, pp. 39-142.

[Ca] J. Carlson, Extensions of mixed Hodge structures, Journées de geométrie algébrique d'Angers (1979), 107-127, Sijthoff and Noordhoff.

[C-K-S] E. Cattani, A. Kaplan, W. Schmid, 1. Degeneration of Hodge structures, Ann. of Math. 123 (1986), $457-535$.

2. $L^{2}$ and intersection cohomologies for a polarizable variation of Hodge structure, Inventiones math. 87, 217-252 (1987)

[De1] P. Deligne, Théorie de Hodge II, Publ. Math. IHES, 40 (1972), 5-57.

[De2] P. Deligne, Equations Différentielles à Points Singuliers Réguliers, Lect. Notes in Math 163, Springer-Verlag, 1970.

[De3] P. Deligne, Théorem de Lèfschetz et critères de dégénérescence de suites spectrales, Publ. Math. I.H.E.S. 35 (1969), 107-126.

[E-M] Philippe Elbaz-Vincent and Stefan Müller-Stach, Milnor $K$-theory of rings, higher Chow groups and applications, Invent. math. 148, (2002), 177-206.

[E-P] H. Esnault and K. H. Paranjape, Remarks on absolute de Rham and absolute Hodge cycles, C. R. Acad. Sci. Paris, t. 319, Série I, (1994), 67-72

[EZ] F. El Zein, Complexe dualisant et applications à la class fondamentale d'un cycle, Bull. Soc. Math. France, Mémoire $\mathbf{5 8}$ (1978).

[F-G] G. Frey and M. Jarden, Approximation theory and the rank of Abelian varieties over large algebraic fields, Proc. London Math. Soc. 28(3), (1974), 112-128.

[Gr] M. Green, Lectures at the CRM Summer School on "The arithmetic and Geometry of Algebraic Cycles", June 7-19, 1998, Banff, Alberta, Canada, (handwritten notes).

[Gr-Gr] M. Green and P. A. Griffiths, Hodge-theoretic invariants for algebraic cycles, International Mathematics Research Notices 2003, No. 9, 477-510.

[G-H] P. A. Griffiths and J. Harris, Principles of Algebraic Geometry, Wiley, New York, 1978.

[Gri] P. A. Griffiths, On the periods of certain rational integrals: I and II, Annals of Math. 90(3), (1969), 460-541.

[Ja] U. Jannsen, Mixed sheaves and filtrations on Chow groups, in: Motives (Editors: U. Jannsen, S. Kleiman, J.-P. Serre), Proceedings of Symposia in Pure Math. 55, 245-302, AMS.

[K] M. Kashiwara and T. Kawai, The Poincaré lemma for variations of polarized Hodge structure, Publ. RIMS, Kyoto Univ. 23 (1987), 345-407.

[KS] T. Katsura and T. Shioda, On Fermat varieties, Tohoku Math. J. 31, (1979), 97-115.

[KO] N. Katz N. and T. Oda, On the differentiation of De Rham cohomology classes with respect to parameters, J. Math. Kyoto Univ., 8 (1968), 199-213.

[Ke1] M. Kerr, Higher Abel-Jacobi maps for 0-cycles. Preprint, 2003.

[Ke2] M. Kerr, Private communication.

[K1] S. Kleiman, Algebraic cycles and the Weil conjectures, Dix exposés sur la cohomologie des schémas, North-Holland, Amsterdam; Masson, Paris, 1968, 359-386.

[K2] S. Kleiman, The standard conjectures, in: Motives (Editors: U. Jannsen, S. Kleiman, J.-P. Serre), Proceedings of Symposia in Pure Math. 55, 3-20, AMS.

[Ki] J. R. King, Log complexes of currents and functorial properties of the Abel-Jacobi map, Duke math. J. Vol. 50, No. 1, (1983), 1-53.

[Le1] J. D. Lewis, A Survey of the Hodge Conjecture, CRM Monograph Series (Amer. Math. Soc.) $10,1999$.

[Le2] J. D. Lewis, A filtration on the Chow groups of a complex projective variety, Compositio Math 128, (2001), 299-322.

[Le3] J. D. Lewis, Cylinder homomorphisms and Chow groups, Math. Nachr. 160, (1993), 205-221.

$[\mathrm{Mu}]$ D. Mumford, Rational equivalence of 0-cycles on surfaces, J. Math. Kyoto Univ., 9 (1969), 195-204.

[R1] A. A. Roitman, Rational equivalence of zero-cycles, Math. USSR Sbornik 18(4) (1972), 571-589.

[R2] A. A. Roitman, On $\Gamma$-equivalence of zero-dimensional cycles, Math. USSR Sbornik 15(4) (1971), 555-567.

[MSa1] M. Saito, Mixed Hodge modules, Publ. RIMS. Kyoto Univ. 26 (1990), 221-333.

[MSa2] M. Saito, Refined cycle maps, in: Algebraic Geometry 2000, Azumino, Advanced Studies in Pure Math. 36 (2002), 115-144, Mathematical Society of Japan, Tokyo. 
[MSa3] M. Saito, On the formalism of mixed sheaves, RIMS preprint.

[MSa4] M. Saito, Direct image of logarithmic complexes, math.AG/0506020.

[RS] A. Rosenschon and M. Saito, Cycle map for strictly indecomposable cycles, Amer. J. Math. 125 (2003), 773-790.

[Sa1] S. Saito, Motives and filtrations on Chow groups, Invent. Math. 125 (1996), 149-196.

[Sa2] S. Saito, Motives and filtrations on Chow groups, II, in: The Arithmetic and Geometry of Algebraic Cycles, Proceedings of the CRM Summer School, June 7-19, 1998, Banff, Alberta, Canada (Editors: B. Gordon, J. Lewis, S. Müller-Stach, S. Saito and N. Yui), NATO Science Series 548 (2000), 321-346, Kluwer Academic Publishers.

[Sa3] S. Saito, Higher normal functions and Griffiths groups, J. of Algebraic Geometry, 11 (2002), 161-201.

[V] C. Voisin, Variations de structure de Hodge et zéro-cycles sur les surfaces générals, Math. Ann., 299 (1994), 77-103.

[S] W. Schmid, Variation of Hodge structure: The singularities of the period mapping, Inventiones Math. 22 (1973), 211-319.

[Sch1] C. Schoen, On Hodge structures and non-representability of Chow groups, Compositio Math. 88 (1993), 285-316.

[Sch2] C. Schoen, Varieties dominated by product varieties, International Journal of Mathematics 7(4) (1996), 541-571.

[So] C. Soulé, Opérations en $K$-théorie algébrique, Canad. J. Math., 37, No. 3, (1985), 488-550.

[Sr] V. Srinivas, Zero cycles on singular varieties, in: The Arithmetic and Geometry of Algebraic Cycles, Proceedings of the CRM Summer School, June 7-19, 1998, Banff, Alberta, Canada (Editors: B. Gordon, J. Lewis, S. Müller-Stach, S. Saito and N. Yui), NATO Science Series 548 (2000), 347-382, Kluwer Academic Publishers.

[Z] S. Zucker, Hodge theory with degenerating coefficients: $L_{2}$ cohomology in the Poincaré metric, Ann. Math. 109 (1979), 415-476.

Department of Mathematical Sciences, University of Alberta, Edmonton, Alberta, Canada

E-mail address: lewisjd@gpu.srv.ualberta.ca

Department of Mathematical Sciences, University of Tokyo Komaba, Meguro, Tokyo 153, Japan

E-mail address: sshuji@msb.biglobe.ne.jp 